\title{
Revisão, análise e classificação da literatura sobre o QFD - tipos de pesquisa, dificuldades de uso e benefícios do método
}

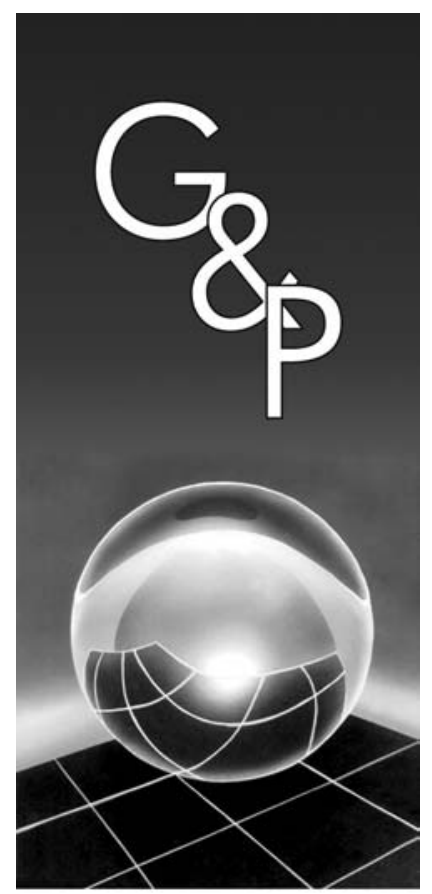

\author{
José Antonio Carnevalli \\ Paulo Augusto Cauchick Miguel
}

Resumo

O objetivo deste trabalho é apresentar uma revisão, análise, classificação e codificação da literatura sobre o QFD. Parte dessa análise é base para o desenvolvimento de um modelo de aplicação do método (QFD) a ser realizado futuramente. As publicações de interesse foram localizadas por meio de consultas nas bases de dados dos periódicos da CAPES, sendo considerados os trabalhos publicados entre 2000 e 2006. As publicações foram classificadas em dois grupos principais: pesquisa conceitual e pesquisa empírica. Utilizou-se o diagrama de afinidades e o diagrama em árvore para organizar os dados da literatura sobre o QFD. As classificações dos artigos e os principais grupos de informações sobre o QFD, organizados pelos diagramas, foram codificados de modo a permitir a visualização de todas as informações de interesse dos artigos. Na classificação das pesquisas, foram encontradas dificuldades na identificação das pesquisas empíricas de "estudo de caso" e "pesquisa-ação", pois, em muitos artigos, havia limitações de informações sobre o método de pesquisa empregado. Os resultados indicam que os trabalhos são publicados de forma dispersa em vários periódicos e que a maioria das publicações estudadas é de cunho teórico-conceitual. Esses trabalhos buscam adaptar o QFD para melhor atender a uma aplicação específica e para aperfeiçoar o método de modo a facilitar a sua aplicação. Sobre os benefícios do uso do método, o que vem se destacando são os benefícios intangíveis relacionados à melhoria do desenvolvimento do projeto de produto. $O$ artigo concluiu que a literatura apresenta diversas dificuldades do uso do QFD, bem como algumas soluções para superar estas dificuldades.

Palavras-chave: Projeto de produto. QFD. Revisão de literatura. Classificação de literatura. Desdobramento da função qualidade.

\section{Introdução}

O QFD (quality function deployment - desdobramento da função qualidade) é um importante método de desenvolvimento de produto, voltado para a tradução de requisitos dos clientes em atividades de desenvolvimento de produtos e serviços. Entretanto, existem várias dificuldades na sua aplicação, dentre as quais: interpretar a "voz do cliente", definir as correlações entre qualidade exigida e características da qualidade (CHAN; WU, 2005); definir a qualidade projetada devido à ambigüidade da qualidade exigida e características da qualidade (RAMASAMY; SELLADURAI, 2004); dificuldade de se trabalhar em equipe; e falta de conhecimento no uso do método (MARTINS; ASPINWALL, 2001). Essas dificuldades têm desmotivado o seu uso. Por esta razão, é importante realizar estudos que busquem entender o QFD, com que objetivos ele vem sendo aplicado, quais os benefícios que tais aplicações trazem e quais são as dificuldades do seu uso para, futuramente, buscar soluções para facilitar a sua aplicação. Para isto, foi realizada uma revisão da literatura de artigos sobre o método. No presente trabalho, são apresentadas revisão, análise e classificação e codificação dos artigos sobre o QFD publicados nos últimos sete anos. O objetivo é verificar quais as características dos estudos realizados, visando identificar possíveis tendências nos estudos sobre o QFD, identificar as técnicas de coleta de dados utilizados, o apoio recebido pelos pesquisadores, dentre outros aspectos. Também foram analisadas as informações que estes trabalhos trazem sobre o uso do método. Nesse sentido, utilizou-se o diagrama de afinidades e o diagrama em árvore para realizar a análise das informações sobre: definições atribuídas ao QFD, ou seja, como os pesquisadores definem o método; pré- 
requisitos do método a serem considerados; dificuldades de aplicação pelo não atendimento dos pré-requisitos necessários para a aplicação do método e que devem ser superadas; benefícios de aplicação, que justificam ou não o seu uso; e, finalmente, recomendações para a aplicação do QFD, que sugerem algumas soluções para algumas das dificuldades de uso do método. A seguir, são apresentados os métodos e as técnicas de pesquisa empregados neste trabalho.

\section{Métodos adotados na presente pesquisa}

Esse trabalho pode ser caracterizado como teóricoconceitual, mais especificamente voltado à busca e revisão da literatura sobre o método QFD. Inicialmente, é importante destacar que para identificar, localizar e adquirir as publicações de interesse, foram consultadas as seguintes bases de dados, disponíveis nos periódicos da CAPES (Coordenação de Aperfeiçoamento de Pessoal de Nível Superior): ACM, ACS, AIP, Balackwell, Cambridge University Pres, Emerald, Gale, HighWire Press, IEEE, Nature, OECD, OVID, Oxford University Press, ProQuest, Sage, SciELO, Science Direct Online e Wilson. A utilização da base de dados disponíveis nos periódicos da CAPES é justificada devido a sua grande abrangência e facilidade de acesso para a maioria dos pesquisadores no Brasil.

Na consulta dos periódicos, buscou-se, como palavrachave, o termo QFD. Realizou-se a busca nos abstracts e, quando esta primeira opção não estava disponível na base consultada, realizou-se a busca do termo QFD no texto completo. O recorte temporal do estudo considerou artigos publicados entre 2000 e 2006, dos quais foram adquiridos os textos de acesso livre. Neste estudo, utilizou-se um horizonte de análise de 7 anos, devido à existência de artigos relevantes anteriores a 5 anos e para verificar de forma mais abrangente possíveis tendências nos estudos sobre o QFD. Inicialmente, foram identificados 254 trabalhos. Entretanto, verificou-se que 14 apenas citavam o termo QFD e 11 eram sobre quantum flavor dynamics, quotient finite dimensional ou quantum fluid dynamics usando a mesma sigla QFD. Esses artigos foram então desconsiderados.

Para a análise dos dados, foram considerados os artigos em periódicos, por terem sido publicados após seleção e avaliação mais criteriosa que os artigos de congressos e simpósios. Foram desconsideradas as análises de resumos de livros, dissertações e teses, pelo fato da base de dados dos periódicos da CAPES divulgar um número limitado de teses e dissertações, não correspondendo ao grande número de defesas que ocorre anualmente sobre o assunto no País. No final, foram analisados 157 artigos em periódicos.
Para realizar os fichamentos dos artigos, utilizou-se uma adaptação de uma tabela usada no levantamento de Martín et al. (1999). Os artigos foram classificados em dois grupos: "pesquisa conceitual" e "pesquisa empírica". Foram considerados como pesquisa conceitual os artigos que tinham como objetivo desenvolver trabalhos "teórico-conceituais", realizar "revisão de literatura", "simulação" ou "modelagem teórica". Foram considerados como pesquisa empírica os artigos que tinham como objetivo realizar estudos tipo "survey", "estudo de caso", "pesquisa-ação" ou "pesquisa experimental". Também se verificou qual a filiação dos pesquisadores, o número médio de autores por artigo e a presença de suporte financeiro, para se realizar o estudo, com o objetivo de se verificar o apoio dado à pesquisa sobre o $\mathrm{QFD}$.

A abordagem dos trabalhos também foi classificada em "quantitativa" e "qualitativa" segundo Martín et al. (1999). Nos casos das pesquisas empíricas, também foi estudada a abrangência do estudo ("regional", "nacional" ou "internacional"); unidade de análise ("pessoas", "grupos", "unidade organizacional”, “empresas”); método de coleta de dados ("documentação direta": "questionário", "entrevistas"; "documentos indiretos": "análise documental", "dados públicos", "dados da imprensa", "bibliográfico"), período analisado somente para estudos de casos conforme Voss et al. (2002) ("retrospectiva", "atual", "longitudinal"). Nas pesquisas conceituais, também foi estudado o método de coleta indireta de dados utilizados ("dados públicos", "dados da imprensa", "bibliográfico"). A classificação dos artigos foi codificada conforme apresentado na Tabela 1, mostrada no Anexo I, para facilitar a visualização dos resultados.

Utilizou-se o diagrama de afinidades e o diagrama em árvore para organizar e agrupar os dados de interesse dos artigos de forma hierárquica, classificando-os de acordo com: o escopo da pesquisa; definições do QFD; dificuldades de aplicação; dificuldade por não atender a pré-requisitos do método; benefícios de uso; pré-requisitos para o uso do método; e, finalmente, recomendações para a aplicação do QFD. O diagrama de afinidades é um método desenvolvido para organizar dados por afinidade, sendo também conhecido como método $\mathrm{Kj}$ de agrupamento (MIZUNO, 1993). Ainda segundo o autor anterior, o diagrama sistemático (também conhecido como diagrama em árvore) organiza os dados na forma de uma árvore e é utilizado para detalhar tarefas e caminhos para atingir os objetivos. No presente trabalho, foi utilizado o diagrama em árvore para organizar os dados já agrupados pelo diagrama de afinidades de forma hierarquizada.

Para permitir a visualização de todas as informações analisadas por artigo estudado, os resultados gerais dos dois diagramas, ou seja, só os principais grupos criados 
pelos diagramas, também foram codificados conforme mostram as Tabelas de 1 a 7 do Anexo I.

A seguir, são apresentados os principais resultados do trabalho, sendo que, no Anexo II, é apresentado um detalhamento dos 157 artigos estudados codificados conforme as Tabelas de 1 a 7.

\section{Resultado da classificação dos artigos estudados}

Com a conclusão da busca bibliográfica, segundo os critérios de busca previamente descritos, foram identificados 157 artigos sobre o QFD, distribuídos em 74 diferentes periódicos referentes ao período de 2000 a 2006. Verificou-se que, em mais de $70 \%$ dos casos, os periódicos apresentavam apenas um artigo sobre o QFD durante os sete anos analisados, mostrando que são raras as publicações sobre o assunto em periódicos, exceto algumas fontes tais como o periódico International Journal of Production Economics que teve oito artigos publicados entre 2000 e 2006 (5,1\% do total) e o periódico International Journal of Quality \& Reability Management com 33 publicações entre 2001 e 2006 (mais de 21\% do total). A Tabela 1 apresenta os treze periódicos que mais publicaram artigos do QFD no período estudado. Dentre eles, destaca-se a Gestão \& Produção como uma das fontes de publicação.

A Figura 1 apresenta o número em porcentagem de publicações por ano. Verifica-se que existe uma certa flutuação no número de artigos, existindo uma queda em 2001 e 2004 com recuperação nos anos de 2003, 2005 e 2006. Uma hipótese, ainda sem comprovação, seria a coincidência de que a maioria das pesquisas estaria em etapas iniciais de desenvolvimento entre os anos de 2001, 2002 e 2004, sem resultados relevantes para a sua publi- cação, os quais só estariam disponíveis em 2003, 2005 e 2006.

Como citado anteriormente, os trabalhos foram classificados em "pesquisa conceitual" ou "pesquisa empírica", sendo que o primeiro tipo prevalece com $67,5 \%$ dos casos, indicando um esforço de vários pesquisadores na busca de adaptar o QFD para melhor atender a uma aplicação específica e, em alguns casos, para melhorar o método de modo a facilitar a sua aplicação.

A Figura 2 classifica mais detalhadamente as pesquisas realizadas nos seguintes grupos: "modelagem"; "teórico-conceitual"; "revisão de literatura"; "simulação"; "survey"; "estudo de caso"; "pesquisa-ação"; e "experimental", sendo que os valores apresentados nas colunas se referem às porcentagens em relação ao total de pesquisa pelo tipo de estudos realizados no período de sete anos. Verifica-se na Figura 2 que a pesquisa "teóricoconceitual" foi a mais realizada no período estudado e, em segundo lugar, se destacam as pesquisas de "estudo de caso" e "pesquisa-ação". Ainda sobre a Figura 2, não foram encontradas pesquisas sobre o QFD classificadas como "modelagem" ou "experimental". Algumas

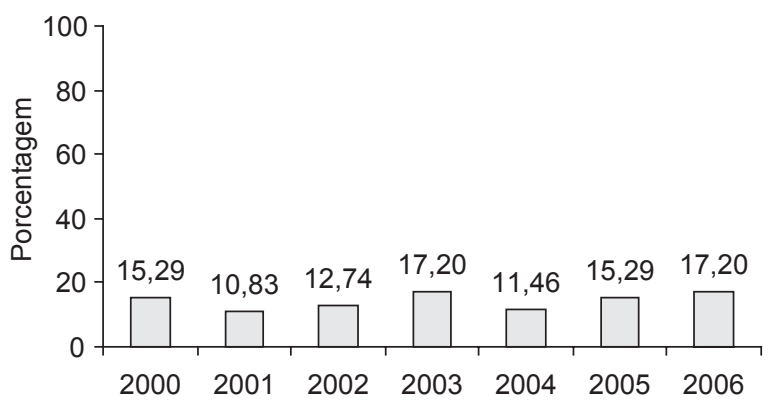

Figura 1. Distribuição percentual do número de publicações por ano.

Tabela 1. Os treze periódicos que mais publicaram artigos sobre QFD.

\begin{tabular}{|c|c|c|c|c|c|c|c|c|}
\hline $\begin{array}{r}\text { Periódicos } \\
\end{array}$ & 2000 & 2001 & 2002 & 2003 & 2004 & 2005 & 2006 & Total \\
\hline Benchmarking: An International Journal & 2 & & & & & 1 & 1 & 4 \\
\hline CE - Concurrent Engineering: Research and Applications & & & & & & 3 & 3 & 6 \\
\hline Computers \& Industrial Engineering & & & 2 & 1 & 1 & 1 & & 5 \\
\hline European Journal of Operational Research & 1 & & 2 & & & & 3 & 6 \\
\hline Gestão \& Produção & & & & & 1 & 1 & 1 & 3 \\
\hline Industrial Management \& Data Systems & & 1 & & 1 & 1 & 1 & & 4 \\
\hline International Journal of Operations \& Production Management & 1 & 1 & 1 & & & & & 3 \\
\hline International Journal of Production Economics & 3 & 1 & 1 & 1 & 1 & & 1 & 8 \\
\hline International Journal of Quality \& Realiability Management & & 2 & 3 & 11 & 8 & 7 & 2 & 33 \\
\hline Journal of Intelligent Manufacturing & 1 & 2 & 1 & & & & & 4 \\
\hline Managerial Auditing Journal & 2 & & 2 & & & & & 4 \\
\hline Managing Service Quality & 2 & & & & 1 & & & 3 \\
\hline Quality Progress & 1 & 1 & & 1 & & 2 & & 5 \\
\hline Total & 13 & 8 & 12 & 15 & 13 & 16 & 11 & 88 \\
\hline
\end{tabular}




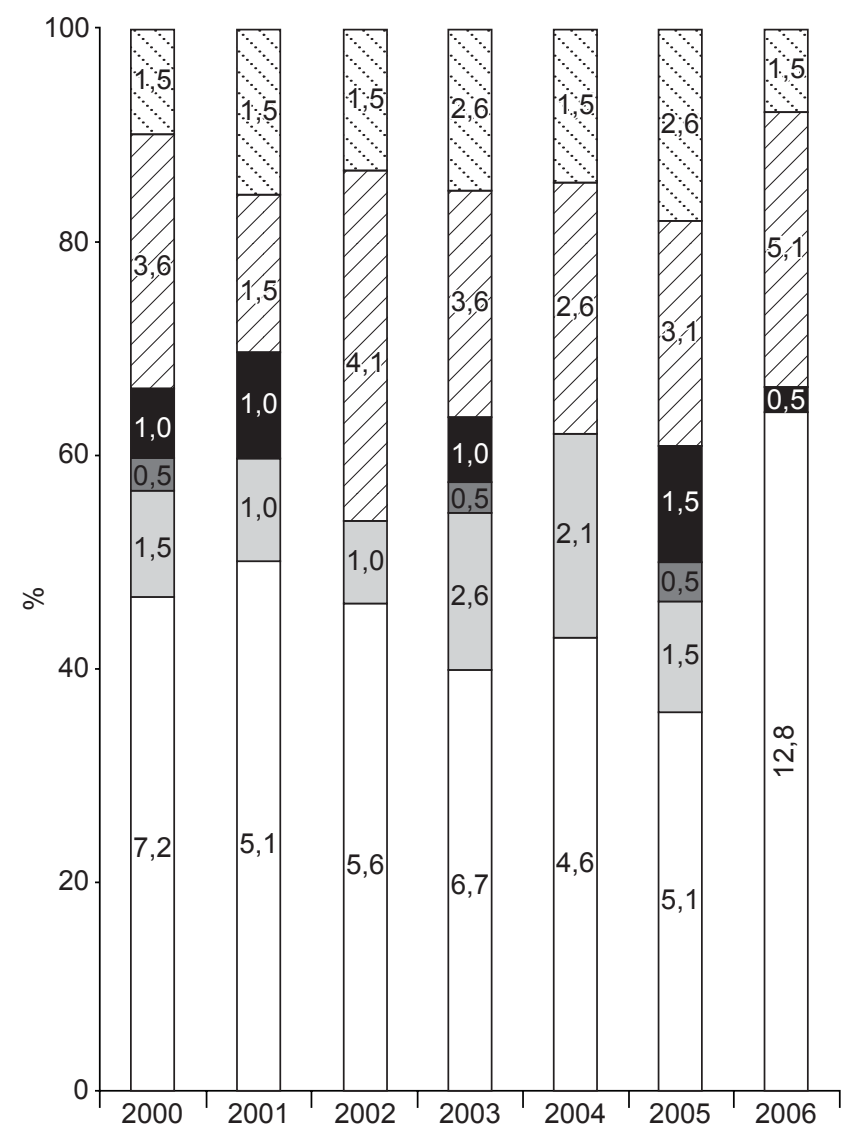

Modelagem $\square$ Teórico-conceitual $\square$ Revisão de literatura
$\square$ Simulação $\square$ Survey $\quad \square$ Estudo de caso
$\square$ Pesquisa-ação $\square$ Experimental

Figura 2. Classificação pelo tipo de estudo realizado.

pesquisas de foco "teórico-conceitual" também foram classificadas como "estudo de caso" por realizarem testes no uso do método em aplicações reais, dentre as quais: Nibbelke et al. (2001), Masui et al. (2003), Yan et al. (2005), Bevilacqua et al. (2006), para citar algumas. Entretanto, por terem o foco do texto predominantemente voltado para o desenvolvimento do método (QFD) na classificação usada ("pesquisa conceitual" ou "pesquisa empírica") foram consideradas como "pesquisa conceitual".

Em relação ao número de pesquisadores por artigos, tem se verificado que, em média, existem dois autores por artigo (média de 2,5), sendo que nos anos de 2000, 2001, 2004 e 2006 a média chega próximo de três autores, o que indica que os estudos são realizados, na maioria dos casos, em equipes, mesmo que sejam pequenas. Em relação à filiação dos pesquisadores, a grande maioria é acadêmica, como esperado, havendo poucas publicações de pesquisadores da indústria. Isto pode significar que as empresas divulgam pouco os seus estudos, já que apenas pouco mais de $21 \%$ dos artigos tem a participação de pesquisadores com vinculação industrial. Também não se tem verificado uma tendência de aumento de publicações desse tipo de filiação (industrial) variando de ano a ano, apresentando uma pequena tendência de queda nos últimos 3 anos. Isso pode indicar uma redução no número de estudos deste tipo na indústria ou uma redução do interesse da empresa em divulgar tais aplicações. Outra característica importante é o perfil dos veículos de divulgação que são, especialmente, acadêmicos. Sobre o apoio financeiro para as pesquisas, somente pouco mais de $20 \%$ dos estudos indicaram algum tipo de apoio. Deste modo, aparentemente, a maioria das pesquisas são realizadas com recursos próprios, seja das empresas que realizam a pesquisa ou recursos já disponíveis nas universidades, não existindo, nos casos observados, um financiamento específico para a pesquisa, com base na descrição dos artigos. Verificou-se que 28\% dos estudos que receberam apoio financeiro eram de Hong Kong, o que pode indicar um maior interesse desta região em desenvolver estudos relacionados ao método.

A Figura 3 apresenta a classificação dos trabalhos em relação às abordagens: "quantitativa" e "qualitativa", sendo que os valores das colunas indicam a porcentagem de ocorrência destas abordagens pelo total de abordagens "quantitativa" e "qualitativa" no período de sete anos. Verificou-se que, com exceção do ano de 2000 e 2003, a abordagem "quantitativa" (quase $51 \%$ dos casos) tem sido mais realizada que as "qualitativas" (pouco mais de $49 \%$ dos casos), isso se deve em alguns casos ao uso crescente de técnicas quantitativas em conjunto com o método QFD como, por exemplo, lógica fuzzy, AHP (analytic hierarchy process), ANP (analytic network process), redes neurais artificiais dentre outras. $\mathrm{O}$ uso destas técnicas tem como objetivo, na maioria dos casos, reduzir a subjetividade das análises realizadas na matriz da qualidade, ou seja, melhorar a operação com o QFD. Com exceção de Cristiano et al. (2001b), que realizaram um estudo quantitativo preditivo, os demais trabalhos são de natureza descritiva, o que era esperado, devido ao grande número de trabalhos conceituais e de estudos que descrevem a aplicação do QFD.

Em relação aos 23 trabalhos empíricos que envolvem ambiente de análise "regional"; "nacional" ou "internacional", estes não apresentaram resultados conclusivos variando de ano a ano. Sobre a unidade de análise destas pesquisas ("pessoas"; "grupos"; "unidade organizacional"; "empresas"), a maioria estudou a "unidade organizacional" (uma empresa), com exceção de 2005 em que se destacou o estudo de múltiplas empresas como unidades de análise. Este resultado é devido ao fato de que a maioria das pesquisas empíricas era estudo de caso único ou pesquisa-ação.

Sobre como os dados foram coletados para desenvolver o método QFD e para a realização das pesquisas, seja por "documentação direta" (“questionário"; "entrevistas") ou 


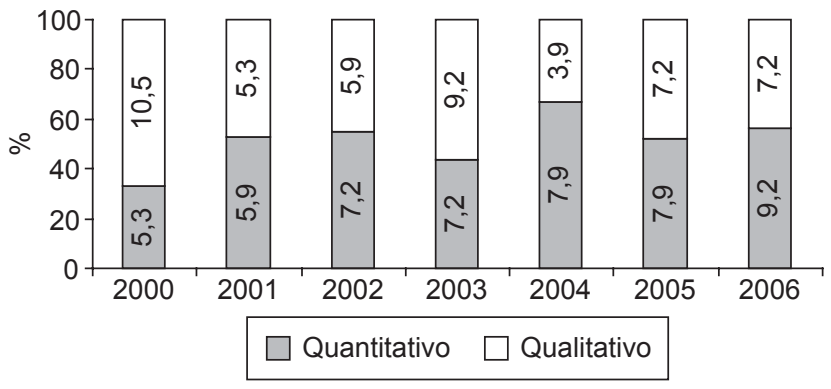

Figura 3. Características dos artigos quanto às abordagens.

"documentação indireta" ("análise documental”; "dados públicos"; "imprensa"; "bibliográfica”), verificou-se que o uso de fontes bibliográficas é o mais utilizado ( $40 \%$ dos casos) e, em segundo lugar, destaca-se o uso de "entrevistas" para a coleta de dados (quase $27 \%$ dos casos). Considerando apenas os trabalhos empíricos, destaca-se o uso de "questionário" (com mais de 30 dos casos).

As pesquisas classificadas como "estudo de caso" também foram classificadas pelo período analisado. Verificou-se, como esperado, que o estudo tipo "atual" (estudo de casos que acompanha uma aplicação contemporânea de um método) é o mais freqüente com mais de 91\% dos casos estudados; seguido de "retrospectiva" (estudos de casos que verificam com dados históricos os fatores de sucesso e fracasso do uso de um método realizado no passado) com 5,4\% dos casos. Sobre os estudos de casos "longitudinais" (que analisa a aplicação de métodos ao longo do tempo), foi encontrado apenas um trabalho, sendo que o estudo envolvia a análise de vários métodos de desenvolvimento de produtos além do QFD. Deste modo, verifica-se que existe uma carência de estudos de casos "longitudinais", que são muito importantes para analisar como a maturidade no uso do QFD influencia nos benefícios e nas dificuldades de aplicação do método.

\section{Análise dos dados sobre o método QFD}

Com o uso do diagrama de afinidades e o diagrama em árvore, foi possível organizar e agrupar de forma hierárquica as informações sobre o escopo das pesquisas, para analisar as definições do QFD, os benefícios, as dificuldades, e as recomendações e pré-requisitos do uso do QFD.

Sobre os objetivos ou escopo das pesquisas estudadas, verificou-se que mais de 53\% dos casos são sobre aplicações do QFD, seja para ajudar a desenvolver estratégia (12\%); produtos (10\%); serviços (14\%), dentre outros. Em alguns destes casos, o QFD é adaptado ou aplicado em conjunto com outros métodos para melhor atender a um objetivo específico. Em mais de $46 \%$ dos casos, o escopo da pesquisa está relacionado com o estudo sobre o método QFD, sejam pesquisas gerais sobre como o QFD está sendo aplicado no mundo e comparar sua eficiência com outros métodos (7\% dos casos) ou estudos para melhorar ou resolver dificuldades do QFD (quase $40 \%$ dos casos).

Sobre como os pesquisadores definem o QFD, foram identificadas cerca de 90 definições, sendo que alguns autores apresentam mais de uma definição no corpo do texto dos trabalhos (seja no abstract, introdução ou conclusão), mas de forma complementar. Depois da organização destes dados, verificou-se que em mais de 33\% dos casos, estas definições estavam limitadas à descrição da matriz da qualidade ou às atividades e objetivos definidos nesta matriz. A matriz da qualidade é responsável por identificar as necessidades dos clientes, definir as prioridades, traduzir estas necessidades em especificações de projeto e definir os valores destas especificações para satisfazer as necessidades dos clientes. Deste modo, esta matriz é muito importante, sendo a mais utilizada e, na maioria das aplicações, a única realizada (MIGUEL, 2003; MARTINS; ASPINWALL, 2001; CRISTIANO et al., 2000), o que explica ser ela usada para definir o método do QFD. Entretanto, a definição do método enfatizando apenas a relação entre requisitos de clientes e características da qualidade pode estar influenciando os usuários do QFD a não considerarem a elaboração de outras matrizes ao aplicar o método. Em mais de 24\% dos casos, a definição do QFD envolve, além da matriz da qualidade outros desdobramentos, como transferir as necessidades dos clientes ( $\mathrm{QE}$ - qualidade exigida) para as várias etapas de projeto do produto e não somente em especificações de projeto (CQ - características da qualidade). Somente em pouco mais de $13 \%$ dos casos, os artigos apresentam uma definição completa do método, que envolve além dos vários desdobramentos realizados nas matrizes do QFD, a definição de procedimento de trabalho a ser realizado durante o desenvolvimento do projeto e fabricação.

Sobre os benefícios da aplicação do QFD, foram identificadas 253 citações que, depois da organização dos dados, foram agrupadas nos seguintes grupos e subgrupos:

Benefícios tangíveis (em pouco mais de $20 \%$ do total de citações):

a) benefícios tangíveis referentes à melhoria do projeto (mais de $16 \%$ das citações), tais como: "melhoria da confiabilidade" (DEVADASAN et al., 2006); "redução do número de alteração do projeto"; "diminuição do tempo de projeto (RAMASAMY; SELLADURAI, 2004) e de seus custos" (HERRMANN et al., 2006); dentre outros; e

b) benefícios tangíveis fora do projeto (3,6\% das citações), tais como: "aumento da receita" (KARSAK 
et al., 2002); "redução de reclamações" (MARTINS; ASPINWALL, 2001); dentre outros.

Benefícios intangíveis (em quase $80 \%$ das citações):

a) benefícios intangíveis referentes à melhoria na condução do projeto (em pouco mais de $72 \%$ dos casos), tais como: "ser uma ferramenta flexível" (LOWE; RIDGWAY, 2000a); "traduzir a QE em CQ" (BENNER et al., 2003); "melhorar a comunicação" (DELANO et al., 2000); "auxiliar na tomada de decisão e definir prioridade" (PARTOVI; CORREDOIRA, 2002); "criar equipes multifuncionais" (LEE; KO, 2000); "aumentar e preservar o conhecimento da empresa" (LAGER, 2005); dentre outros; e

b) benefícios intangíveis fora do projeto (em quase 8,0\% dos casos), tais como: "aumento da satisfação dos clientes" (KUMAR et al., 2006).

Deste modo, verifica-se que a maioria dos benefícios da aplicação do QFD, apresentados na literatura com base na amostra consultada, são intangíveis e relacionados à melhoria na condução do projeto, de modo que as empresas devem iniciar a aplicação do método considerando este fato, pois os benefícios tangíveis podem ocorrer ou não com a aplicação do QFD.

Sobre os pré-requisitos do QFD, foram identificadas 56 citações sobre o assunto. Destas, 12 consideram a necessidade do apoio da alta administração (mais de 20\% dos casos). Depois da organização dos dados pelo diagrama de afinidades e pelo diagrama em árvore, verificou-se que os demais pré-requisitos estão indiretamente ligados ao apoio da alta administração tais como: "fornecer suporte à equipe do QFD" (ERDER; PURER, 2003); "fornecer recursos necessários" (CRISTIANO et al., 2001b); "desenvolver uma equipe capacitada" (KENGPOL, 2004); "realizar o treinamento necessário de todos os envolvidos"; "ter a empresa um certo nível de qualidade: controle de processo e solução de problemas de modo sistemático" (GOVERS, 2001); dentre outros. Deste modo, os pré-requisitos estão relacionados a aspectos externos ao método do QFD, muitos deles comuns nas aplicações de métodos complexos.

$\mathrm{Na}$ análise da literatura foram identificadas 56 citações sobre "dificuldades externas ao QFD", relacionadas pelo não atendimento dos pré-requisitos do QFD, mostrando a importância de se atender a estes pré-requisitos. As dificuldades mais citadas foram: "a falta de apoio da alta administração" (GINN; ZAIRI, 2005); "falta de recursos" (KENGPLO, 2004); e "falta de conhecimento sobre o QFD” (MARTINS; ASPINWALL, 2001); dentre outras.

Sobre as dificuldades metodológicas de aplicação do QFD, foram identificadas 113 citações, sendo a mais citada: "dificuldades devido ao grande tamanho das matrizes" (DIKMEN et al., 2005). Com a organização dos dados pelo diagrama de afinidades e pelo diagrama em árvore, verificou-se que as principais dificuldades metodológicas do QFD estão relacionadas às etapas de elaboração da matriz da qualidade (quase $80 \%$ das citações) tais como: "interpretar a voz do cliente" (GINN; ZAIRI, 2005); "identificar os requisitos dos clientes mais importantes" (YAN et al., 2005); "realizar tomada de decisão no projeto, por não serem claras as correlações entre os requisitos" (FUNG et al. 2006). Deste modo, verifica-se que reduzir as dificuldades metodológicas da elaboração da matriz da qualidade é um fator-chave para incentivar e ampliar o uso do QFD.

Foram identificadas 194 citações sobre recomendações para auxiliar a aplicação do QFD, além de introduzir métodos e ferramentas para reduzir as dificuldades relacionadas ao seu uso. Realizou-se uma codificação dos principais grupos definidos com o apoio do diagrama de afinidades e do diagrama em árvore. Com esta análise verificou-se que as principais recomendações citadas nos artigos referem-se ao desdobramento da qualidade, e relacionam-se principalmente à construção da matriz da qualidade (mais de $65 \%$ dos casos). Em relação às principais dificuldades citadas anteriormente, as recomendações identificadas foram as seguintes:

Para reduzir o tamanho da matriz da qualidade: identificar as principais qualidades exigidas (QEs) e somente estas farão parte da matriz (MARSOT, 2005). Lowe e Ridgway (2000a) chegam a sugerir um número máximo de 8 itens para QEs e para CQs (Características da Qualidade). Entretanto, aqueles autores não analisam se uma matriz da qualidade 8 por 8 seria suficiente para se desenvolver projetos complexos, como muitas vezes o QFD é utilizado. Para ajudar na identificação das QE prioritárias, Chien e Su (2003) utilizam um índice nacional de satisfação dos clientes. Chou (2004) propõe uma outra estratégia para diminuir a matriz: ao invés de reduzir o número de itens na matriz da qualidade, os usuários deveriam dividir o projeto em grupos de subprojetos, de modo a analisar os dados separadamente em várias matrizes pequenas. Entretanto, tal abordagem pode gerar novas dificuldades já que não considera as correlações entre CQs vs. CQs, no caso de se dividir a matriz em subprojetos. Com uma estratégia semelhante, Shin e Kim (2000) apresentam um método de reorganização dos dados da matriz da qualidade em grupos separados utilizando um algoritmo de agrupamento. Do mesmo modo que no caso anterior, tal abordagem pode gerar novas dificuldades a serem consideradas antes da aplicação do QFD. Isto porque, ao desfazer a organização de dados realizada anteriormente na elaboração das tabelas da $\mathrm{QE}$ e da CQ com um algoritmo de agrupamento, pode dificultar a verificação de falhas na elaboração destas tabelas (por exemplo, mistura de dados do $2^{\circ}$ nível no $3^{\circ}$ nível), as quais seriam identificadas durante as correlações. 
Em relação à dificuldade gerada por falta de apoio da alta administração, não foi encontrada uma recomendação que diretamente lidasse com o problema, pois esta é uma questão relacionada à conscientização dos usuários e se a alta gerência acredita ou não no método. Entretanto, indiretamente foram identificadas as seguintes recomendações: Cristiano et al. (2001b) comprovam que o apoio da alta administração influencia positivamente na melhoria do processo e nos produtos que estão usando o QFD. Politis (2003) também verificou que o estilo e o tipo de liderança afetam a aplicação do QFD, recomendando um estilo que incentive participação e tomada de decisões, mostrando que além do apoio da administração o tipo de liderança também deve ser considerado.

Sobre a dificuldade de falta de recursos também não existe uma solução direta na literatura estudada, pois ou ela está relacionada à falta de apoio administrativo (já discutido anteriormente) ou à condição financeira da empresa. Para este segundo caso, fica válida a recomendação de Lowe e Ridgway (2000a) de buscar adaptar o QFD à realidade da empresa e aos objetivos do projeto.

Sobre a dificuldade de falta de conhecimento no QFD, três das cinco recomendações identificadas foram: "realizar treinamento prático do QFD", "utilizar o treinamento para reduzir as resistências ao uso do QFD", e "realizar treinamento sobre entendimento das necessidades dos clientes" (GINN; ZAIRI, 2005); nos casos em que as empresas já têm aplicações anteriores do QFD, estas devem ser usadas para o treinamento (CRISTIANO et al., 2001b).

Em relação à dificuldade de "interpretar a voz do cliente", a literatura apresenta várias alternativas para ajudar a resolver esta dificuldade, dentre elas: o uso da lógica fuzzy (SHIPLEY et al., 2004); uso de grupo focal com brainstorming e diagrama de causa e efeito (CHOU, 2004); uso do modelo de Kano (TAN; SHEN, 2000); dentre outras.

Para ajudar na "definição da qualidade projetada" a literatura recomenda: o uso de lógica fuzzy (CHEN; WENG, 2006; CHEN; WENG, 2003) ou o método de Taguchi e Rede Neural (BOUCHEREAU; ROWLANDS, 2000a). Também é recomendado: utilizar o ANP (analytic network process) com o ZOGP (Zero - One Programming) (KARSAK et al., 2002), utilizar o MADM modelo (multi-attribute decision-making) (HAN et al., 2004), usar o QFD em conjunto com DA (decisision analysis) para facilitar na tomada de decisão (DELANO et al., 2000); dentre outros.

Uma tendência que tem aparecido como recomendação nas pesquisas do QFD, a ser verificada e considerada é a utilização da lógica fuzzy, de forma isolada ou com outros métodos e ferramentas, dentro da matriz da qualidade, para definir a QE (qualidade exigida) (SHIPLEY et al., 2004); priorizar ou identificar o grau de importância da
QE (BÜYÜKÖZHAN; FEYZIOGLU, 2005); realizar os cálculos desta matriz (CHAN; WU, 2005); ajudar nas correlações (BOUCHEREAU; ROWLANDS, 2000a) e na definição da qualidade projetada (CHEN; WENG, 2003).

Outra tendência identificada é a utilização do método AHP (analytic hierarchy process) com ou sem a lógica fuzzy para ajudar a definir o grau de importância da qualidade exigida (MYINT, 2003) e para ajudar nas correlações entre os dados das matrizes (PARTOVI, 2001; 2006). Na seqüência, são apresentadas uma análise crítica, dificuldades encontradas e as conclusões deste estudo.

\section{Análise crítica e dificuldades encontradas no estudo}

Com a análise dos 157 artigos sobre o QFD, verificou-se a flexibilidade de aplicação do método, o qual não se limita ao desenvolvimento de produtos, processos e serviços, se mostrando um excelente método de análise e correlação de informações. Por exemplo, o método tem se mostrado útil para desenvolver estratégias em educação (LEE; LO, 2003), ajudar a definir a localização de centros de distribuição de mercadorias (CHUANG, 2002), avaliar e selecionar fornecedores (BEVILACQUA et al., 2006), dentre outras aplicações. Apesar desta flexibilidade de aplicação, a maioria dos estudos tem limitado o uso do QFD à elaboração da matriz da qualidade, o que pode limitar os seus resultados. As adaptações realizadas para atingir um objetivo específico do estudo ou para melhorar o método têm focado principalmente nesta matriz, na qual se encontram as principais dificuldades de elaboração. Entretanto, existem poucos estudos que apresentam recomendações para a melhoria do treinamento do QFD, para orientar na elaboração do modelo conceitual, no desdobramento da confiabilidade, apresentar metodologias para ajudar a resolver gargalos de engenharia ou na avaliação da aplicação do QFD, aspectos que também geram dificuldades no uso do método. A quase inexistência de estudo de caso longitudinal em QFD, durante o período analisado, indica uma importante lacuna na análise do método, pois tal estudo permite verificar como a maturidade no uso do QFD influencia nos seus benefícios e na diminuição ou não das suas dificuldades de utilização. Incentivar estudos de caso longitudinais e que busquem soluções não limitadas apenas à elaboração da matriz da qualidade são fatores importantes para a contínua evolução do QFD.

Em relação à classificação das pesquisas, foram encontradas dificuldades na identificação das pesquisas empíricas tipo "estudo de caso" e "pesquisa-ação", por restrições nas informações sobre os métodos empregados e por alguns destes estudos se caracterizarem por uma classificação diferente da definida pelos autores. Alguns 
destes artigos são exemplificados a seguir. Leprevost e Mazur (2005) e Gerst (2004) definem suas pesquisas como "estudo de caso", mas como há indicações da participação destes pesquisadores na aplicação estes estudos foram classificados como "pesquisa-ação". No caso de Omachonu e Barach (2005), os pesquisadores não definiram se realizaram um "estudo de caso" ou "pesquisa-ação" e, como o artigo não deixa claro se houve participação dos autores na aplicação, a pesquisa foi classificada como "estudo de caso".

\section{Conclusões}

A identificação de 157 artigos sobre o QFD distribuídos em 74 diferentes periódicos nas bases de dados do portal da CAPES, indicou a existência de dispersão nas publicações sobre o assunto, pois mais de $54 \%$ dos artigos estavam distribuídos em 63 diferentes periódicos. Em muitos desses periódicos, foi identificada apenas uma publicação sobre o QFD durante o período avaliado de 7 anos. Mesmo nos sete periódicos que mais publicaram sobre o QFD, quatro deles têm menos de sete artigos sobre o assunto neste período. Conclui-se então uma certa dispersão no uso do método.

Pode-se concluir, em relação ao tipo de pesquisa realizada, que a maior parte é conceitual e descritiva, utilizando principalmente fonte de dados bibliográficos. O objetivo dos trabalhos, na maioria dos casos, era adaptar o QFD para uma aplicação específica. Também se destacam os estudos que buscavam melhorar o método com a introdução de outras ferramentas e técnicas. Entretanto, na grande parte dos casos, as aplicações são limitadas à matriz da qualidade, o que pode reduzir os resultados do método. Na classificação dos trabalhos, foram encontradas dificuldades na identificação das pesquisas empíricas tipo "estudo de caso" e "pesquisa-ação", pois em muitos artigos havia limitações de informações sobre os métodos adotados e, em alguns casos, havia falhas nas definições feitas pelos autores dos artigos, considerando como "estudo de caso" pesquisas que tinham características de "pesquisa-ação". A carência de estudo de caso longitudinal, indica a quase inexistência de dados atuais de como a maturidade no uso do QFD afeta a sua aplicação, mostrando a necessidade de incentivar tais estudos.
O uso do diagrama de afinidades e do diagrama em árvore se mostrou eficiente para organizar e agrupar as informações de interesse bibliográfico sobre o QFD, a serem consultadas durante o desenvolvimento de um sistema de aplicação do QFD, próxima etapa do presente trabalho. Com estes diagramas, verificou-se que os benefícios intangíveis do QFD relacionados com a melhoria do projeto são os mais citados, provavelmente por serem os mais freqüentes na aplicação. Também foi possível não somente verificar as principais dificuldades de aplicação do QFD, como quais soluções já existem na literatura para resolvê-las. Verifica-se que os estudos estão mais focados em resolver dificuldades na matriz da qualidade, o que era esperado, pois as principais dificuldades estão relacionadas a esta matriz. Entretanto, poucos estudos foram realizados para resolver outros aspectos importantes, os quais também devem ser estudados, como melhorar o treinamento no método. Sobre as definições do QFD, estas estão limitadas, na maioria dos casos, a aspectos da matriz da qualidade, provavelmente por ser a mais importante para o projeto e a mais utilizada, como já apresentado em outros estudos (CRISTIANO et al., 2000; MIGUEL, 2003). Pode-se considerar que esta definição pode estar limitando o uso do QFD apenas em uma matriz. A codificação das classificações das pesquisas e dos principais agrupamentos definidos pelos diagramas permitiu uma rápida visualização de vários aspectos dos artigos estudados, como por exemplo, tipo de estudo e abordagem utilizada, escopo dos artigos, definições do QFD, benefícios e dificuldades identificadas nos estudos sobre o uso do QFD.

Duas tendências identificadas, que serão futuramente avaliadas, são o uso da lógica fuzzy para realizar a maioria das etapas da matriz da qualidade, resolvendo dificuldades de análises e priorização realizadas dentro destas matrizes, e a utilização do método AHP com ou sem a lógica fuzzy para ajudar a definir o grau de importância da qualidade exigida e para ajudar nas correlações entre os dados das matrizes. Entretanto, é necessário verificar se tais aplicações não deixariam o QFD ainda mais complexo, desmotivando mais o seu uso. As próximas etapas do trabalho compreendem o desenvolvimento de um modelo de aplicação do QFD, que considere a redução das dificuldades de uso do QFD. 


\title{
Literature review, classification, and analysis on QFD - types of research, difficulties of the use, and benefits of the method
}

\begin{abstract}
This paper shows a QFD literature review as well as its classification, codification, and analysis. Part of this study is the basis for the development of a systemic model of QFD application to be used in the future. Scientific publications were obtained from CAPES data base considering studies published from 2000 to 2006. The papers were classified into conceptual research and empirical research. The affinity diagram and the tree diagram were used to organize the QFD literature data. The classification of the papers and the main groups of information about the QFD, organized according to the diagrams, were codified, so the information needed could be found. Some difficulties were encountered when classifying the research such as identifying empirical research, e.g. "case study" or "action research" because, most of the times, there was limited information about the research method employed. The results show that the papers were published scatteredly in several different journals, and that the majority of them have a theoretical/conceptual characteristic. These studies are aimed at adapting QFD to a specific application and to improve the method in order to facilitate its use. The use of this method has provided intangible benefits related to the improvement of the product development project. The article demonstrated that the literature presents many difficulties of using QFD although it presents some solutions to overcome those difficulties.
\end{abstract}

Keywords: Product project. QFD. Literature review. Literature classification. Quality function deployment.

\section{Referências bibliográficas}

AL-MASHARI, M.; ZARI, M.; GINN, D. Key enablers for the effective implementation of QFD: a critical analysis. Industrial Management \& Data Systems, v. 105, n. 9, p. 1245-1260, 2005.

AKAO, Y.; MAZUR, G. H. The leading edge in QFD: past, present and future. International Journal of Quality \& Reliability Management, v. 20, n. 1, p. 20-35, 2003.

BENNER, M.; LINNEMANN, A. R.; JONGEN, W. M. F.; FOLSTAR, P. Quality Function Deployment (QFD) - can it be used to develop food products? Food Quality and Preference, v. 14, n. 4, p. 327-339, 2003.

BEVILACQUA, M.; CIARAPICA, F. E.; GIACCHETTA, G. A fuzzy-QFD approach to supplier selection. Journal of Purchasing and Supply Management, v. 12, n. 1, p. 14-27, 2006.

BIER, I. D.; CORNESKY, R. Using QFD to construct a higher education curriculum. Quality Progress, v. 34, n. 4, p. 64-68, 2001.

BOOYSEN, G. J.; BARNARD, L. J.; TRUSCOTT, M.; BEER, D. J. Anaesthetic mouthpiece develooment through QFD and customer interaction with functional prototypes. Rapid Prototyping Journal, v. 12, n. 4, p. 189-197, 2006.

BOSCH, V. G; ENRÍQUEZ, F. T. TQM and QFD: exploiting a customer complaint management system. International Journal of Quality \& Reliability Management, v. 22, n. 1, p. 30-37, 2005.

BOTTANI, E.; RIZZI, A. Strategic management of logistics service: A fuzzy QFD approach. International Journal of Production Economics, v. 103, n. 2, p. 585-599, 2006.

BOUCHEREAU; V.; ROWLANDS, H. Methods and techiniques to help quality function deployment (QFD). Benchmarking: An International Journal, v. 7, n. 1, p. 8-16, 2000a.

. Quality function deployment: the unused tool. Engineering Management Journal, v. 10, n. 1, p. 45-52, 2000 b.
BÜYÜKÖZHAN, G.; FEYZIOGLU, O. Group decision making to better respond customer needs in software development. Computers \& Industrial Engineering, v. 48, n. 2. p. 427-441, 2005.

CARNEVALLI, J. A.; SASSI, A. C.; MIGUEL, P. A. C. Aplicação do QFD no desenvolvimento de produtos: levantamento sobre seu uso e perspectivas para pesquisas futuras. Gestão \& Produção, v. 11, n. 1, p. 33-49, 2004.

CHAN, L. K.; WU, M. L. Quality function deployment: A literature review. European Journal of Operational Research, v. 143, n. 3, p. 463-497, 2002.

A systematic approach to quality function deployment with a full illustrative example. Omega - The International Journal of Management Science, v. 33, n. 2, p. 119-139, 2005.

CHAO, L. P.; ISHII, K. Project quality function deployment. International Journal of Quality \& Reliability Management, v. 21, n. 9, p. 938-958, 2004.

CHEN, L. H.; WENG, M. C. A fuzzy model for exploiting quality function deployment. Mathematical And Computer Modelling. v. 38, n. 5-6, p. 559-570, 2003.

An evaluation approach to engineering design in QFD processes using fuzzy goal programming models. European Journal of Operational Research, v. 172, n. 1, p. 230-248, 2006.

CHEN, Y.; FUND, R. Y. K. Rating technical attributes in fuzzy QFD by integrating fuzzy weighted average method and fuzzy. European Journal of Operational Research, v. 174, n. 3, p. 1553-1566, 2006.

CHENG, L. C. QFD in product development: methodological characteristics and a guide for intervention. International Journal of Quality \& Reliability Management, v. 20, n. 1, p. 107-122, 2003.

CHIEN, T. K.; SU, C. T. Using the QFD concept to resolve customer satisfaction strategy decisions. International Journal of Quality \& Reliability Management, v. 20, n. 3, p. 345-359, 2003. 
CHIN, K. S.; PUN, K. F.; LEUNG, W. M.; LAU, H. A quality function deployment approach for improving technical library and information services: a case study. Library Management, v. 22 , n. 4/5, p. 195-204, 2001.

CHOU, S. M. Evaluating the service quality of undergraduate nursing education in Taiwan - using quality function deployment. Nurse Education Today, v. 24, n. 4, p. 310-318, 2004.

CHUANG, P. T. A QFD approach for distribution's location model. International Journal of Quality \& Reliability Management, v. 19, n. 8/9, p. 1037-1054, 2002.

CLARGO, M. The designer organisation: Organisations too can benefit from the application of design and quality tools, and with startling results! International Journal of Quality \& Reliability Management, v. 21, n. 9, p. 973-983, 2004.

COELHO, A. M.; MOURÃO, A. J. F.; PEREIRA, Z. L. Improving the use of QFD with Axiomatic Design. CE Concurrent Engineering: Research and Applications, v. 13, n. 3, p. 233-239, 2005.

COSTA, A. I. A.; DEKKER, M.; JORGE, W. M. F. Quality function deployment in the food industry: a review. Food Science $\boldsymbol{\&}$ Technology, v. 11, n. 9-10, p. 306-314, 2001.

CRISTIANO, J. J.; LIKER, J. K.; IIIWHITE, C. C. Customer-driven product development through quality function deployment in the U.S. and Japan. Journal of Product Innovation Management, v. 17, n. 4, p.286-308, 2000.

Application of Multiattribute Decision Analysis to Quality Function Deployment for Target Setting. IEEE Transactions on Systems, Man, and Cybernetics, v. 31, n. 3, p. 366-382, 2001a. Key factors in the successful application of quality function deployment (QFD). IEEE Transactions on Engineering Management, v. 48, n. 1, p. 81-95, 2001b.

DELANO, G.; PARNELL, G. S.; SMITH, C.; VANCE, M. Quality function deployment and decision analysis: A R\&D case study. International Journal of Operations \& Production Management, v. 20, n. 5, p. 591-609, 2000.

DEVADASAN, S. R; KATHIRAVAN, N.; THIRUNAVUKKARASU, V. Theory and practice of total quality function deployment: A perpective from a traditional pump-manufacturing environment. The TQM Magazine, v. 18, n. 2, p. 143-161, 2006.

DIKMEN, I.; BIRGONUL, M. T.; KIZILTAS, S. Strategic use of quality function deployment (QFD) in the construction industry. Building and Environment, v. 40, n. 2, p. 245-255, 2005.

DIJKSTRA, L.; BIJ, H. V. D. Quality function deployment in healthcare: Methods for meeting customer requirements in redesign and renewal. International Journal of Quality \& Reliability Management, v. 19, n. 1, p. 67-89, 2002.

DRIVA, H.; PAWAR, K. S.; MENON, U. Measuring product development performance in manufacturing organizations. International Journal of Production Economics, v. 63, n. 2, p. 147-159, 2000.

DUFFUAA, S. O.; AL-TURKI, U; HAWSAWI, F. M. Quality and Reliability corner Quality function deployment for designing a basic statistics course. International Journal of Quality \& Reliability Management, v. 20, n. 6, p. 740-750, 2003.

DUHOVNIK, J.; KUSAR, J.; TOMAZEVIC, R.; STARBEK, M. Development Process with Regard to Customer Requirements. Concurrent Engineering: Research and Applications, v. 14, n. 1, p. 67-82, 2006.

ELDIN, N. A Promising Planning Tool: Quality Function Deployment. Cost Engineering, v. 44, n. 3, p. 28-37, 2002.
ENRÍQUEZ, F. T.; OSUNA, A. J.; BOSCH, V. G. Prioristising customer needs at spectator events. International Journal of Quality \& Reliability Management, v. 21, n. 9, p. 984-990, 2004.

ERDER, M.; PURER, P. QFD in the Architecture Development Process. IT Professional, v. 5, n. 6, p. 44-52, 2003.

EROL, I.; FERRELL JR., W. G. A methodology for selection problems with multiple, conflicting objectives and both qualitative and quantitative criteria. International Journal of Production Economics, v. 86, n. 3, p. 187-199, 2003.

FERNANDES, J. M.; REBELATO, M. G. Proposta de um método para integração entre QFD e FMEA. Gestão \& Produção, v. 13, n. 2, p. 245-259, 2006.

FEHLMANN; T. M. Strategic management by business metrics: An application of combinatory metrics. International Journal of Quality \& Reliability Management, v. 1, n. 1, p. 134-145, 2003.

The impact of linear algebra on QFD. International Journal of Quality \& Reliability Management, v. 22, n. 1, p. 83-96, 2005.

FRANCESCHINI, F.; ROSSETTO, S. QFD: an interactive algorithm for the prioritization of product's technical design characteristics. Integrated Manufacturing Systems, v. 13, n. 1, p. 69-75, 2002.

FUNG, R. Y. K.; CHEN, Y.; TANG, J. Estimating the functional relationships for quality function deployment under uncertainties. Fuzzy Sets and Systems, v. 157, n. 1, p. 98-120, 2006.

GANDHINATHAN, R.; RAVISWARAN, N.; SUTHAKAR, M. QFD- and VE-enabled target costing: a fuzzy approach. International Journal of Quality \& Reliability Management, v. 21, n. 9, p. 1003-1011, 2004.

GERST, R.M. QFD in large-scale social system redesign. International Journal of Quality \& Reliability Management, v. 21, n. 9, p. 959-972, 2004.

GINN, D.; ZAIRI, M. Best practice QFD application: an internal/ external benchmarking approach based on Ford Motors' experience. International Journal of Quality \& Reliability Management, v. 22, n. 1, p. 38-58, 2005.

GONZÁLEZ; M. E.; QUESADA, G.; MUELLER, R.; MORAMONGE, C. A. QFD strategy house: an innovative tool for linking marketing and manufacturing strategies. Marketing Intelligence \& Planning, v. 22, n. 3, p. 335-348, 2004a.

GONZÁLEZ, M. E.; QUESADA, G.; PICADO, F.; ECKELMAN, C.A. Customer satisfaction using QFD: an e-banking case. Managing Service Quality, v. 14, n. 4, p. 317-330, 2004b.

GONZÁLEZ, M. E.; QUESADA, G.; MACK, R.; URRUTIA, I. Building an activity-based costing hospital model using quality function deployment and benchmarking. Benchmarking: An International Journal, v. 12, n. 4, p. 310-329, 2005.

GOVERS, C. P. M. QFD not just a tool but a way of quality management. International Journal of Production Economics, v. 69, n. 2, p. 151-159, 2001.

GOULD, L. S. QFD analysis: from customer needs to design specs. Automotive Design \& Production, v. 118, n. 6, p. 56-57, 2006.

GUNASEKARAN, N.; RAVISWARAN, N.; SUTHAKAR, M. Optimizing supply chain management using fuzzy approach. Journal of Manufacturing Technology Management, v. 17, n. 6, p. 737-749, 2006.

HALOG, A.; SCHULTMANN, F.; RENTZ, O. Using quality function deployment for technique selection for optimum environmental performance improvement. Journal of Cleaner Production, v. 9, n. 5, p. 387-394, 2001. 
HAN, C. H.; KIM, K. J.; CHOI, S. H. Prioritizing engineering characteristics in quality function deployment with incomplete information: A linear partial ordering approach. International Journal of Production Economics, v. 91, n. 3, p. 235-249, 2004.

HAN, S. B.; CHEN, S. K.; EBRAHIMPOUR, M.; SODHI, M. S. A conceptual QFD planning model. International Journal of Quality \& Realiability Management, v. 18, n. 8, p. 796-812, 2001.

HANUMAIAH, N.; RAVI, B.; MUKHERJEE, N. P. Rapid hard tooling process selection using QFD-AHP methodology. Journal of Manufacturing Technology, v. 17, n. 3, p. 332-350, 2006.

HERRMANN, A.; HUBER, F.; BRAUNSTEIN, C. Market-driven product and service design: Bridging the gap between customer needs, quality management, and customer satisfaction. International Journal of Production Economics, v. 66, n. 1, p. 77-96, 2000.

HERRMANN, A.; HUBER, F.; ALGESHEIME, R.; TOMCZAK, T. An empirical study of quality function deployment on company performance. International Journal of Quality \& Reliability Management, v. 23, n. 4, p. 345-366, 2006.

HERZWURM, G.; SCHOCKERT, S. The leading edge in QFD for software and electronic business. International Journal of Quality \& Reliability Management, v. 20, n. 1, p. 36-55, 2003.

HSIAO, S. W; LIU, E. A structural component-based approach for designing product family. Computers in Industry, v. 56, n. 1, p. 13-28, 2005.

HUANG, G. Q.; MAK, K.L. Synchronous quality function deployment (QFD) over world wide web. Computers \& Industrial Engineering, v. 42, n. 2-4, p. 425-431, 2002.

HUNT, R. A.; XAVIER, F. B. The leading edge in strategic QFD. International Journal of Quality \& Reliability Management, v. 20, n. 1, p. $56-73,2003$.

HWARNG, H.B.; TEO, C. Translating customers' voices into operations requirements: A QFD application in higher education. International Journal of Quality \& Reliability Management, v. 18, n. 2, p. 195-225, 2001.

IRANMANESH, S. H.; THOMSON, V.; SALIMI, M. H. Design Parameter Estimation using a Modified QFD Method to Improve Customer Perception. CE - Concurrent Engineering: Research and Applications, v. 13, n. 1, p. 57-67, 2005.

JALHAM, I. S.; ABDELKADER, W. T. Improvement of organizational efficiency and effectiveness by developing a manufacturing strategy decision support system. Business Process Management Journal, v. 12, n. 5, p. 588-607, 2006.

JOHNSON, C. N. QFD Explained: use this process to ensure quality throghout the product development process. Quality Progress, v. 36, n. 3, p. 104, 2003.

JUSSEL, R.; ATHERTON, M. How QFD improves product development across sites. Quality Focus, v. 4, n. 1, p. 28-33, 2000.

KAHRAMAN, C.; ERTAY, T.; BÜYÜKÖZKAN, G. A fuzzy optimization model for QFD planning process using analytic network approach. European Journal of Operational Research, v. 171, n. 2, p. 390-411, 2006.

KARSAK, E. E.; SOZER, S.; ALPTEKIN, S. E. Product planning in quality function deployment using a combined analytic network process and goal programming approach. Computers \& Industrial Engineering, Great Britain, v. 44, n. 1, p. 171-190, 2002.

KARSAK, E. E. Fuzzy multiple objective programming framework to prioritize design requirements in quality function deployment. Computers \& industrial Engineering, v. 47, n. 2-3, p. 149-163, 2004.

KENGPOL, A. Quality Function Deployment (QFD) in Small to Medium-sized Enterprises: A Study of Obstacles in Implementing QFD in Thailand. International Journal of Management, v. 21, n. 3, p. 393-402, 2004.

KILLEN, C. P.; WALKER, M.; HUNT, R. A. Strategic planning using QFD. International Journal of Quality \& Reliability Management, v. 22, n. 1, p. 17-29, 2005.

KIM, S. H.; JANG, D. H.; LEE, D. H.; CHO, S. H. A methodology of constructing a decision path for IT investment. Journal of Strategic Information Systems, v. 9, n. 1, p. 17-38, 2000a.

KIM, K. J.; MOSKOWITZ, H.; DHINGRA, A.; EVANS, G. Fuzzy multicriteria models for quality function deployment. European Journal of Operational Research, v. 121, n. 3, p. $504-518,2000$ b.

KRIEG, R. Impact of structured product definition on market success. International Journal of Quality \& Reliability Management, v. 21, n. 9, p. 991-1002, 2004.

KUMAR, R.; MIDHA, P. S. A QFD based methodology for evaluating a company's PDM requirements for collaborative product development. Industrial Management \& Data Systems, v. 101, n. 3, p. 126-131, 2001.

An objective approach for identifying the strategic components of a PDM (Product data management) system. Industrial Management \& Data Systems, v. 104, n. 1, p. 56-67, 2004.

KUMAR, A.; ANTONY, J.; DHAKAR, T. S. Integrating quality function deployment and benchmarking to achieve greater profitability. Benchmarking: An International Journal, v. 13, n. 3, p. 290-310, 2006.

KUTUCUOGLU, K. Y.; HAMALI, J.; IRANI, Z. A framework for managing maintenance using performance measurement systems. International Journal of Operations \& Production Management, v. 21, n. 1/2, p. 173-194, 2001.

KWONG, C. K.; BAI, H. A fuzzy AHP approach to the determination of importance weights of customer requirements in quality function deployment. Journal of Intelligent Manufacturing, v. 13, n. 5, p. 367-377, 2002.

Determining the importance weights for the customer requirements in QFD using a fuzzy AHP with an extent analysis approach. IIE Transactions, v. 35, n. 7, p. 619-626, 2003.

LAGER, T. The industrial usability of quality function deployment: a literature review and synthesis on a meta-level. R\&D Management, v. 35, n. 4, p. 409-426, 2005.

LEE, S. F.; LO, K. K.; LEUNG, R. F.; KO, A. S. O. Strategy formulation framework for vocational education: integrating SWOT analysis, balanced scorecard, QFD methodology and MBNQA education criteria. Managerial Auditing Journal, v. 15, n. 8 , p. 407-423, 2000.

LEE, S. F.; KO, A. S. O. Building balanced scorecard with SWOT analysis, and implementing "Sun Tzu's The Art of Business Management Strategies" on QFD methodology. Managerial Auditing Journal, v. 15, n. 1/2, p. 68-76, 2000.

LEE, S. F.; LO, K. K. e-Enterprise and mangement course development using strategy formulation framework for vocational education. Journal of Materials Processing Technology, v. 139, n. 1-3, p. 604-612, 2003.

LEPREVOST, J.; MAZUR, G. Quality infrastructure improvement: using QFD to manage project priorities and project management resources. International Journal of Quality \& Reliability Management, v. 22, n. 1, p. 10-16, 2005. 
LI, D.; MCKAY, A.; PENNINGTON, A. DE; BARNES, C. A Web-based tool and a heuristic method for cooperation of manufacturing supply chain decisions. Journal of Intelligent Manufacturing, v. 12, n. 5-6, p. 433-453, 2001.

LIM, P. C.; TANG, N. K. H. The development of a model for total quality healthcare. Managing Service Quality, v. 10, n. 2, p. 103-111, 2000.

LIN, S. J; WEI, C. C. A Study on the Linear Programming in Time Cost Analysis of Product Improve Design- a Focus on Computer Mouse Products. Journal of American Academy of Business, v. 7, n. 2, p. 182-186, 2005.

LIN, W. T.; HUNG, Y. H.; HUANG, C. T.; WU, C. C. A Performance Evaluation of the After-Sales Service Information Systems provided by Taiwanese Machine Tool Industry. International Journal of Management, v. 22, n. 1, p. 112-126, 2005.

LIN, M. C.; WANG, C. C.; CHEN, T. C. A Strategy for Managing Customer-oriented Product Design. Concurrent Engineering, v. 14, n. 3, p. 231-244, 2006.

LIU, X. F. Software quality function deployment. IEEE Potentials, v. 19, n. 5, p. 14-16, 2000.

LORENZO, S.; MIRA, J.; OLARTE, M.; GUERRERO, J.; MOYANO, S. Análisis matricial de la voz del cliente: QFD aplicado a la gestión sanitaria. Gaceta Sanitaria, v. 18, n. 6, p. 464-471, 2004

LOWE, A.; RIDGWAY, K.; ATKINSON, H. QFD in new production technology evaluation. International Journal of Production Economics, v. 67, n. 2, p. 103-112, 2000.

LOWE, A.; RIDGWAY, K. UK user's guide to quality function deployment. Engineering Management Journal, v. 10, n. 3, p. 147-155, 2000a.

. Optimization Impossible? : The importance of customer segmentation in quality function deployment. Quality Progress, v. 33, n. 7, p. 59-64, 2000b.

LUNSFORD, P.; MONROE, R.; PAGLIARI, L. A Method for Defining a Relative Measure of Strategic Importance and Operational. Review of Electronic and Industrial Distribution, v. 5, n. 1, p. 32-45, 2006.

MARCOS, S. K; JORGE, J. T. Desenvolvimento de tomate de mesa, com o uso do método QFD (Desdobramento da Função Qualidade), comercializado em um supermercado. Horticultura Brasileira, v. 20, n. 3, p. 490-496, 2002.

MARSOT, J. QFD: a methodological tool for integration of ergonomics at the design stage. Applied Ergonomics, v. 36, n. 2, p. 185-192, 2005.

MARTÍN, L. Á. G.; CABESTRE, F. J. R.; VEGA, A. V. R. El estado actual de la investigación empírica sobre economía de la empresa: análisis de las publicaciones españlas. Papeles de economía española, n. 78-79, p. 302-317, 1999.

MARTINS, A.; ASPINWALL, E. M. Quality function deployment: an empirical study in the UK. Total Quality Management, v. 12, n. 5 , p. 575-588, 2001.

MASUI, K.; SAKAO, T.; KOBAYASHI, M.; INABA, A. Applying Quality Function Deployment to environmentally conscious design. International Journal of Quality \& Reliability Management, v. 20, n. 1, p. 90-106, 2003.

MATSUDA, L. M.; ÉVORA, Y. D. M.; BOAN, F. S. O Método Desdobramento da Função Qualidade - QFD - no planejamento do serviço de enfermagem. Revista Latino-Americana de Enfermagem. Enfermagem, v. 8, n. 5, p. 97-105, 2000.

MIGUEL, P. A. C. The state-of-the-art of the Brazilian QFD applications at the top 500 companies. International Journal of Quality \& Reliability Management, v. 20, n. 1, p. 74-89, 2003.
. Evidence of QFD best practices for product development: a multiple case study. International Journal of Quality \& Reliability Management, v. 22, n. 1, p. 72-82, 2005.

MIGUEL, P. A. C.; TELFESER, M.; MARUCA, A.; GALLONETTI, A.; SARACURA, A.; MARTINS, L.; HORI, M.; RIBEIRO, P.; CAMPOS, R. D.; MARCONATO, T.; MORA, V. Desdobramento da Qualidade no Desenvolvimento de filmes flexíveis para embalagens, Polímeros: Ciência e Tecnologia, v. 13, n. 2, p. 87-94, 2003.

MILAN, M.; BARROS, J. W. D.; GAVA, J. L. Planning soil tillage using Quality Function Deployment (QFD). Scientia Agricola, v. 60 , n. 2, p. 217-221, 2003.

MIZUNO, S. Gerência para melhoria da qualidade: As Setes Novas Ferramentas de Controle da Qualidade, Rio de Janeiro: LTC - Livros Técnicos e Científicos Ed., 1993. 282 p.

MOORES, B. M. Radiation safety management in health care - The application of Quality Function Deployment. Radiography, v. 12, n. 4, p. 291-304, 2006.

MYINT, S. A framework of an intelligent quality function deployment (IQFD) for discrete assembly environment. Computers \& Industrial Engineering, v. 45, n. 2, p. 269-283, 2003.

NIBBELKE, R.; FERRO, D.; HOOGEBOOM, P. Design and Evaluation with the Human in Mind. AIR \& Space Europe, v. 3, n. 3-4, p. 218-220, 2001.

NOGUEIRA, J. C. A budgeting method using Quality Function Deployment. The Engineering Economist, v. 48, n. 4, p. 333-344, 2003.

OLEWNIK, A. T.; LEWIS, K. On Validating Engineering Design Decision Support Tools. CE - Concurrent Engineering: Research and Applications, v. 13, n. 2, p. 111-122, 2005.

OLHAGER, J.;WEST, B. M. The house of flexibility: using the QFD approach to deploy manufacturing flexibility. International Journal of Operations \& Production Management, v. 22, n. 1 , p. 50-79, 2002.

OMACHONU, V.; BARACH, P. QFD in a managed care organization. Quality Progress, v. 38, n. 11, p. 36-41, 2005.

PARKIN, N.; LINSLEY, M. J.; CHAN, J. F. L.; STEWARDSON, D. J. The introduction of QFD in a UK original equipment manufacturer. Managerial Auditing Journal, v. 17, n. 1, p. 43-54, 2002.

PARTOVI, F. Y. An analytic model to quantify strategic service vision. International Journal of Service Industry Management, v. 12, n. 5, p. 476-449, 2001.

An analytic model for locating facilities strategically. OMEGA: The International Journal of Management Science, v. 34, n. 1, p. 41-55, 2006.

PARTOVI, F. Y.; CORREDOIRA, R. A. Quality function deployment for the good of soccer. European Journal of Operational Research, v. 137, n. 3, p. 642-656, 2002.

PINTO, M. Data representation factors and dimensions from the quality function deployment (QFD) perspective. Journal of Information Science, v. 32, n. 2, p. 116-130, 2006.

PISZCZALKI, M. QFD: The low-tech way to collaborate. Automotive Design \& Production, v. 115, n. 1, p. 16-17, 2003.

PIZZOLATO, M.; CATEN, C. S.T.; FOGLIATTO, F. S. Definiçãodo prazo de garantia de um produto otimizado experimentalmente. Gestão \& Produção, v. 12, n. 2, p. 239-235, 2005.

POLITIS, J. D. QFD: the role of various leadership styles. Leadership \& Organization Development Journal, v. 24, n. 4, p. 181-192, 2003. 
QFD, organisational creativity and productivity. International Journal of Quality \& Reliability Management, v. 22, n. 1, p. 59-71, 2005.

PRAMOD, V. R.; DEVADASAN, S. R.; MUTHU, S.; JAGATHYRAJ, V. P.; MOORTHY, G. D. Methodology And theory integrating TPM and QFD for improving quality in maintenance engineering. Journal of Quality in Maintenance Engineering, v. 12, n. 2, p. 150-171, 2006.

PRESLEY, A.; SARKIS, J.; LILES, D. H. A Soft-Systems Methodology Approach for Product and Process Innovation. IEEE Transactions on Engineering Management, v. 47, n. 3, p. 379-392, 2000.

PULLMAN,M. E.; MOORE, W. L.; WARDELL, D. G. A comparison of quality function deployment and conjoint analysis in new product design. The Journal of Product Innovation Management, v. 19, n. 5, p. 354-364, 2002.

PUN, K. F.; CHIN, K. S.; LAU, H. A QFD/hoshin approach for service quality deployment: a case study. Managing Service Quality, v. 10, n. 3, p. 156-169, 2000.

RAHIM, A. R. A.; BAKSH, M. S. N. Application of quality function deployment (QFD) method for pultrusion machine design planning. Industrial Management \& Data Systems, v. 103, n. 6, p. 373-387, 2003.

RAHIMI, M.; WEIDNER, M. Integrating Design for Environment (DfE) Impact Matrix into Quality Function Deployment (QFD) Process. The Journal of Sustainable Product Design, v. 2, n. 1-2, p. 29-41, 2002.

RAMASWAMY, N. R.; SELLADURAI, V.; GUNASEKARAN, A. Just-in-time implementation in small and medium enterprises. Work Study, v. 51, n. 2, p. 85-90, 2002.

RAMASAMY, N. R.; SELLADURAI, V. Fuzzy logic approach to prioritise enginnering characteristics in quality function deployment (FL-QFD). International Journal of Quality \& Reliability Management, v. 21, n. 9, p. 1012-1023, 2004.

SANFORD, J. How useful is QFD? Quality Progress, v. 38, n. 1, p. 51-59, 2005.

SHARMA, J. R.; SHARMA, D. K.; RAWANI, A. M. Driven Product Development. Manufacturing Engineer, v. 85, n. 3, p. 38-41, 2006.

SHEN, X. X.; TAN, K. C.; XIE, M. An integrated approach to innovative product development using Kano's model and QFD. European Journal of Innovation Management, v. 3, n. 2, p. 91-99, 2000a.

Benchmarking in QFD for quality improvement. Benchmarking: An International Journal, v. 7, n. 4, p. 282-291, 2000b.

The implementation of quality function deployment based on linguistic data. Journal of Intelligent Manufacturing, v. 12 , n. 1 , p. $65-75,2001$.

SHER, S. S. The Application of Quality Function Deployment (QFD) in Product Development - The Case Study of Taiwan Hypermarket Building. The Journal of American Academy of Business, v. 8, n. 2, p. 292-295, 2006.

SHIN, J. S.; KIM, K-J.; CHANDRA, M. J. Consistency check of a house of quality chart. International Journal of Quality \& Reliability Management, v. 19, n. 4, p. 471-484, 2002.

SHIN, J. S.; KIM, K. J. Complexity reduction of a design problem in QFD using decomposition. Journal of Intelligent Manufacturing, v. 11, n. 4, p. 339-354, 2000.

SHIPLEY, M. F.; KORVIN, A. DE; YOON, J-M. Fuzzy quality function deployment: determining the distributions of effort dedicated to technical change. International Transactions in Operational Research, v. 11, n. 3, p. 293-307, 2004.
SILVA, F. L. R.; CAVALCA, K. L.; DEDINI, F. G. Quality and reliability corner combined application of QFD and VA tools in the product design process. International Journal of Quality \& Reliability Management, v. 21, n. 2, p. 231-252, 2004.

SOHN, S. Y.; CHOI, I. S. Fuzzy QFD for supply chain management with reliability consideration. Reliability Engineering and System Safety, v. 72, n. 3, p. 327-334, 2001.

STEHN, L.; BERGSTRÖM, M. Integrated design and production of multi-storey timber frame houses - production effects caused by customer-oriented design. International Journal of Production Economics, v. 77, n. 3, p. 259-269, 2002.

TAN, C. M. Customer-focused build-in reliability: a case study. International Journal of Quality \& Reliability Management, v. 20, n. 3, p. 378-397, 2003.

TAN, K. C.; SHEN, X. X. Integrating Kano's model in the planning matrix of quality function deployment. Total Quality Management, v. 11, n. 8, p. 1141-1151, 2000.

TANG, J.; FUNG, R. Y. K.; XU, B.; WANG, D. A new approach to quality function deployment planning with financial consideration. Computers \& Operations Research, v. 29, n. 11, p. 1447-1463, 2002.

THAKKAR, J.; DESHMUKH, S. G.; SHASTREE, A. Total quality management (TQM) in self-financed technical institutions: A quality function deployment (QFD) and force field analysis approach. Quality Assurance in Education, v. 14, n. 1, p. 54-74, 2006.

TONTINI, G. Deployment of customer needs in the QFD using a modified Kano model. Journal of Academy of Business and Economics, v. 2, n. 1, p. 103-113, 2003.

VOSS, C.; TSIKRIKTSIS, N.; FROHLICH, M. Case Research: case research in operations management. International Journal of Operations \& Production Management, v. 22, n. 2, p. 195-219, 2002.

WALDEN, J. Performance excellence: a QFD approach. International Journal of Quality \& Reliability Management, v. 20, n. 1, p. 123-133, 2003.

WALKER, M. Customer - driven breakthroughs using QFD and policy deployment. Management Decision, v. 40, n. 3, p. 248-256, 2002.

WEI, C. C.; LIU, P. H.; CHEN, C.B. An automated system for product specification and design. Assembly Automation, v. 20, n. 3, p. 225-232, 2000.

XAVIER, F. B.; HUNT, R. A. Strategy in turbulent times. Managerial Auditing Journal, v. 17, n. 1/2, p. 55-59, 2002.

YAN, W.; KHOO, L. P.; CHEN, C. H. A QFD-enabled product conceptualisation approach via design knowledge hierarchy and RCE neural network. Knowledge-Based Systems, v. 18, n. 6, p. 279-293, 2005.

YANG, Y. Q.; WANG, S. Q.; DULAIMI, M.; LOW, S. P. A fuzzy quality function deployment system for buildable design decision-makings. Automation in construction, v. 12, n. 4, p. 381-393, 2003.

YANG, C. C.; LIN, W. T.; LIN, M. Y.; HUANG, J. T. A study on applying FMEA to improving ERP introduction: An example of semiconductor related industries in Taiwan. International Journal of Quality \& Reliability Management, v. 23, n. 3, p. 298-322, 2006.

YUNG, K. L.; KO, S. M.; KWAN, F. Y.; TAM, H. K.; LAM, C. W.; NG, H. P.; LAU, K. S. Application of Function Deployment Model in Decision Making for New Product Development. Concurrent Engineering: Research and Applications, v. 14, n. 3 , p. $257-267,2006$. 


\section{ANEXO I: Tabelas de codificação}

Tabela 1. Codificação da classificação dos artigos sobre o QFD.

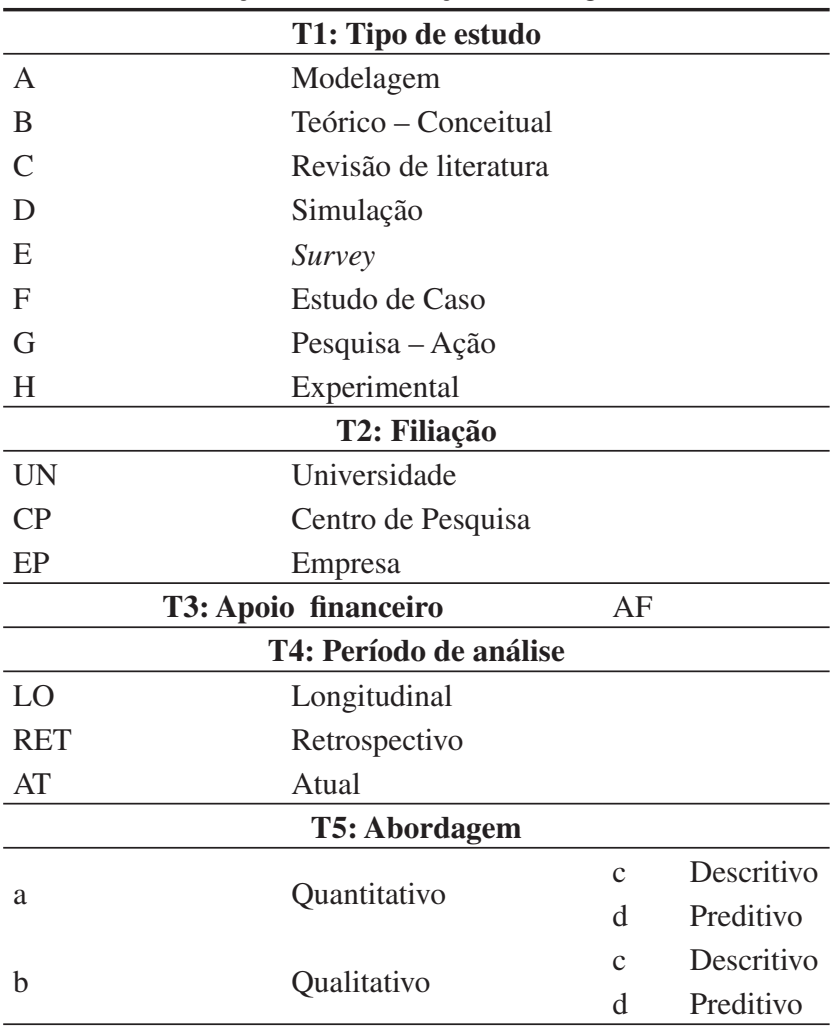

\begin{tabular}{ll}
\hline & T6: Abrangência geográfica \\
RE & Regional \\
NA & Nacional \\
IN & Internacional \\
\hline & T7: Unidade de análise \\
\hline PE & Pessoas \\
GR & Grupos \\
O & Unidade Organizacio- \\
& nal \\
EMP & Empresas \\
\hline & T8: Documentação \\
\hline 1 & Questionário \\
2 & Entrevistas \\
3 & Análise Documental \\
4 & Dados Públicos \\
5 & Imprensa \\
6 & Bibliográfica \\
\hline
\end{tabular}

Tabela 2. Codificação do escopo dos artigos sobre o QFD (T9).

\begin{tabular}{|c|c|}
\hline \multicolumn{2}{|r|}{ Aplicação do QFD } \\
\hline A1 & Uso do QFD para desenvolver estratégia \\
\hline $\mathrm{A} 2$ & $\begin{array}{l}\text { Aplicação para ajudar a implantar método, normas, } \\
\text { etc. }\end{array}$ \\
\hline A3 & Aplicação para desenvolver produtos \\
\hline A4 & Aplicação para desenvolver software \\
\hline A5 & Aplicação para desenvolver serviços \\
\hline A6 & Uso do QFD para ajudar em planejamento \\
\hline A7 & Aplicações diversas \\
\hline \multicolumn{2}{|r|}{ Pesquisa sobre o QFD } \\
\hline A8 & Pesquisa sobre o uso do QFD num país ou em vários. \\
\hline A9 & Comparar o QFD com outros métodos \\
\hline \multicolumn{2}{|r|}{$\begin{array}{c}\text { Estudo para melhorar ou resolver } \\
\text { dificuldades do uso do QFD }\end{array}$} \\
\hline A10 & Pesquisa sobre o QFD para ajudar na implantação \\
\hline A11 & $\begin{array}{l}\text { Pesquisa sobre o QFD para identificar os fatores de } \\
\text { sucesso }\end{array}$ \\
\hline A12 & $\begin{array}{l}\text { Para melhorar ou resolver dificuldades do uso do } \\
\text { QFD, aplicação de método e ferramentas no QFD }\end{array}$ \\
\hline
\end{tabular}

Tabela 3. Codificação das definições do QFD e benefícios apresentados nos artigos (T10).

\begin{tabular}{cl}
\hline & \multicolumn{1}{c}{ T10: Definição } \\
\hline b1 & Matriz da qualidade \\
b2 & Outras matrizes além da matriz da qualidade \\
b3 & Outras matrizes além da matriz da qualidade e des- \\
& dobramento do trabalho \\
b4 & Ferramenta de planejamento multifuncional \\
b5 & Técnica de gestão \\
b6 & O QFD é parte do TQM \\
b7 & Outras definiç̃os do QFD \\
\hline \multicolumn{2}{c}{ T11: Benefícios do QFD } \\
\hline Benefícios tangíveis referentes à melhoria do projeto \\
\hline B1 & Melhorar a confiabilidade \\
B2 & Reduzir alterações no projeto \\
B3 & Reduzir o tempo \\
B4 & Reduzir os custos \\
B5 & Outros benefícios tangíveis referentes à melhoria do \\
& projeto \\
B6 & Benefícios Tangíveis fora do projeto \\
\hline Benefícios Intangíveis referentes à melhoria do projeto \\
\hline B7 & Método flexível \\
B8 & Melhoria da comunicação \\
B9 & Ajudar na análise dos dados e na tomada de decisão \\
& racional \\
B10 & Trabalho em equipe \\
B11 & Melhorar o know how e a sua conservação \\
B12 & Integração de ferramentas e métodos \\
B13 & Outros benefícios intangíveis referentes à melhoria \\
& do projeto \\
B14 & Benefícios intangíveis fora do projeto \\
\hline
\end{tabular}


Tabela 4. Codificação dos pré-requisitos do uso do QFD (T12).

\section{Pré-requisito}

C0 Pré-requisito: necessidade de ter suporte da alta administração

C1 Necessidade de suporte e recursos para o QFD

C1A Pré-requisito para desenvolver uma boa equipe

C1B Pré-requisito: necessidade de conhecer o QFD antes de aplicá-lo

C1C Pré-requisito definir o mercado

Tabela 5. Codificação das dificuldades por não atender a pré-requisitos do uso do QFD (T13).

\section{Dificuldades externas do uso do QFD}

DP1 Dificuldade por falta de apoio da alta administração

DP11 Dificuldade por falta de recursos

DP111 Dificuldade: Falta de conhecimento no QFD

DP112 Dificuldade: Falta de tempo para o projeto

DP2 Dificuldade por falta de foco no projeto

DP3 Dificuldade: identificar a necessidade do cliente

DP4 Dificuldade por falta de comprometimento da equipe do QFD

DP5 Dificuldade pela falta de conhecimento do tipo de produto a ser desenvolvido

DP6 Dificuldade: devido à estrutura da empresa

Tabela 6. Codificação das dificuldades metodológicas do uso do QFD (T14).
Dificuldades metodológicas do QFD

D1 Dificuldade: Fazer as matrizes

D1A Dificuldade: Fazer a tabela de qualidade exigida

D1B Dificuldade de desdobrar os requisitos dos clientes em características da qualidade

D1C Dificuldade: Definir a qualidade projetada D1C1 Dificuldade tamanho da matriz da qualidade

D2 Dificuldade gerada pelo tipo de produto a ser desenvolvido D3 Outras dificuldades ou dúvidas de aplicação

\begin{tabular}{cc}
\hline \multicolumn{2}{c}{ Dificuldades metodológicas do QFD } \\
\hline D1 & Dificuldade: Fazer as matrizes \\
& D1A $\quad$ Dificuldade: Fazer a tabela de qualidade exigida \\
& D1B $\quad$ Dificuldade de desdobrar os requisitos dos clientes \\
& em características da qualidade \\
& D1C Dificuldade: Definir a qualidade projetada \\
& D1C1 Dificuldade tamanho da matriz da qualidade \\
\hline D2 & Dificuldade gerada pelo tipo de produto a ser desenvolvido \\
\hline D3 & Outras dificuldades ou dúvidas de aplicação \\
\hline
\end{tabular}

Tabela 7. Codificação das recomendações para diminuir dificuldades do uso do QFD (T15).

\begin{tabular}{|c|c|c|c|}
\hline \multicolumn{4}{|c|}{ Recomendações para diminuir dificuldades do uso do QFD } \\
\hline \multirow[t]{5}{*}{ E1 } & \multicolumn{3}{|c|}{ Preparar-se para usar o QFD } \\
\hline & \multirow[t]{4}{*}{ E1A } & Algum & as recomendações para implantação \\
\hline & & E1A1 & Recomendações para a administração \\
\hline & & E1A2 & Recomendações sobre a equipe do QFD \\
\hline & & E1A3 & Recomendações sobre o treinamento \\
\hline \multirow[t]{14}{*}{ E2 } & \multicolumn{3}{|c|}{ Fazer o QD } \\
\hline & \multirow[t]{8}{*}{$\mathrm{E} 2 \mathrm{~A}$} & Matriz & da qualidade \\
\hline & & $\mathrm{E} 2 \mathrm{~A} 1$ & Coletar e analisar as vozes dos clientes \\
\hline & & E2A2 & Grau de importância da QE \\
\hline & & E2A3 & Definição da qualidade planejada \\
\hline & & E2A4 & Extração das características de qualidade \\
\hline & & E2A5 & Diminuir o tamanho da matriz da qualidade \\
\hline & & E2A6 & Fazer as correlações do QFD \\
\hline & & E2A7 & Definição da qualidade projetada \\
\hline & E2B & Elabora & ar o modelo conceitual \\
\hline & \multirow[t]{4}{*}{$\mathrm{E} 2 \mathrm{C}$} & $\begin{array}{l}\text { Recom } \\
\text { mento }\end{array}$ & $\begin{array}{l}\text { endações para o desdobramento e preenchi- } \\
\text { das tabelas e matrizes }\end{array}$ \\
\hline & & $\mathrm{E} 2 \mathrm{C} 1$ & Desdobramento das Funções \\
\hline & & $\mathrm{E} 2 \mathrm{C} 2$ & $\begin{array}{l}\text { Resolver gargalos de engenharia e problemas } \\
\text { de confiabilidade }\end{array}$ \\
\hline & & E2C3 & Desdobramento dos Custos \\
\hline E3 & \multicolumn{3}{|c|}{ Recomendações para revisar a aplicação do QFD } \\
\hline $\mathrm{E} 4$ & \multicolumn{3}{|c|}{ Outras recomendações } \\
\hline
\end{tabular}




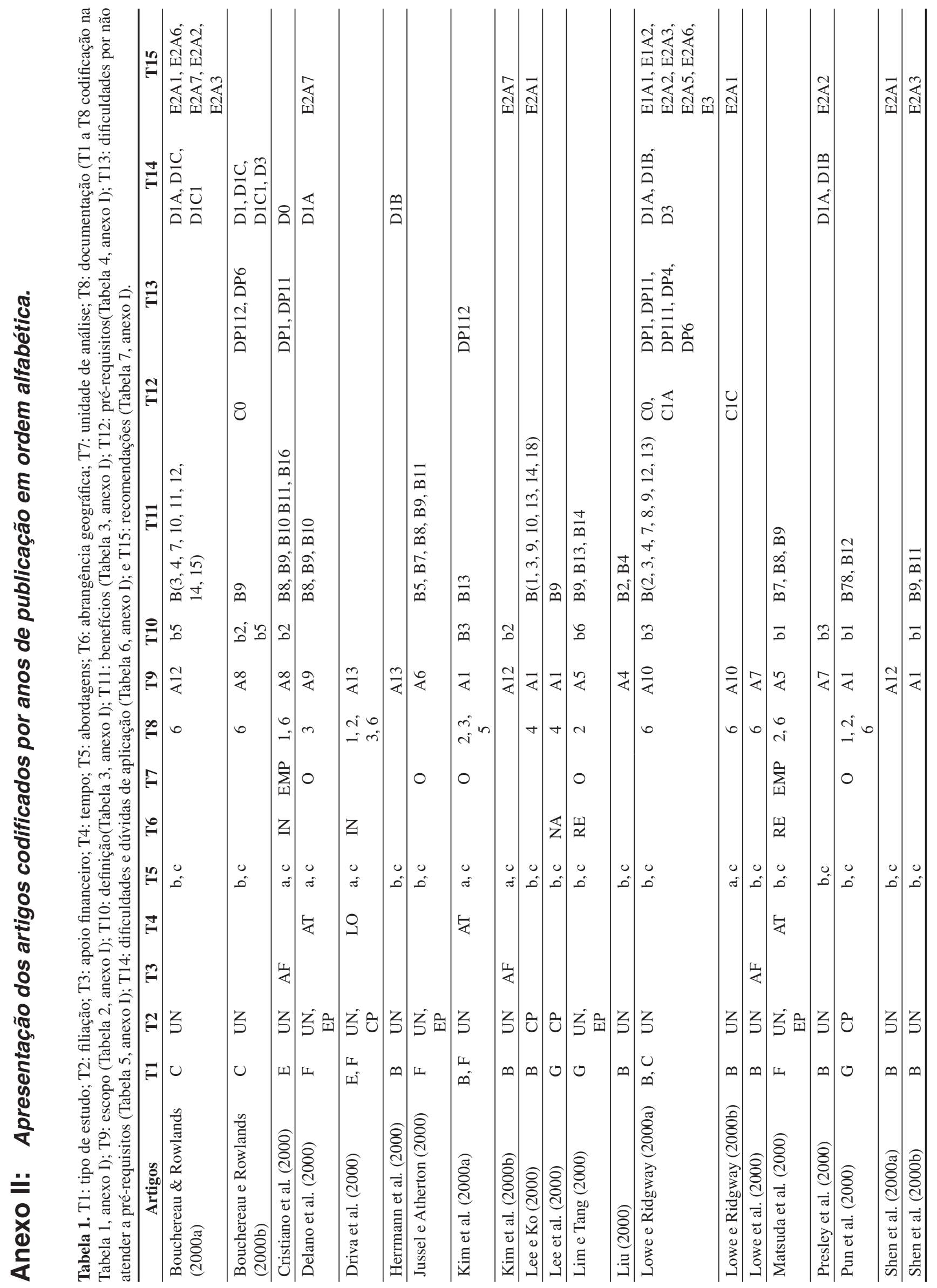




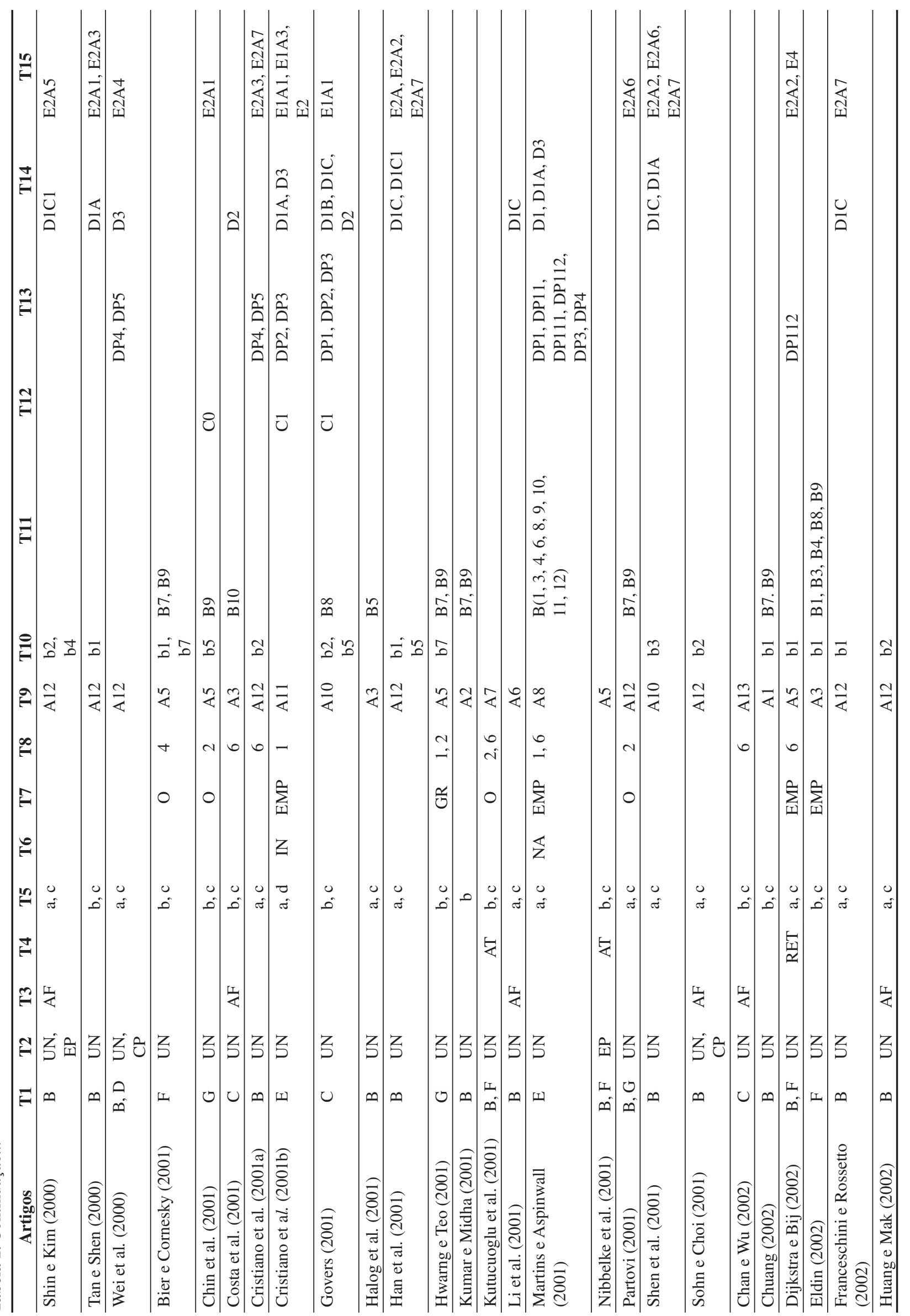




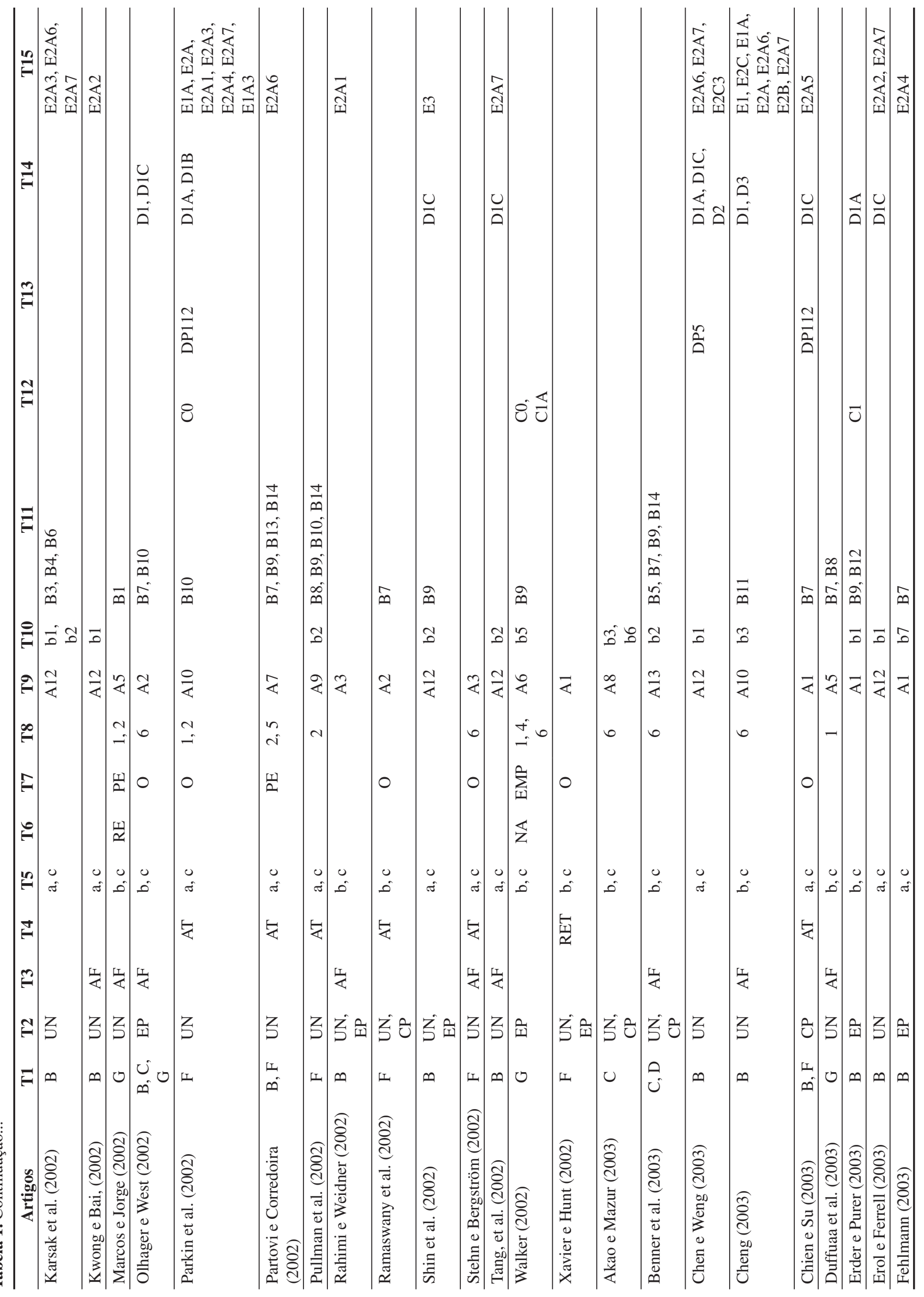




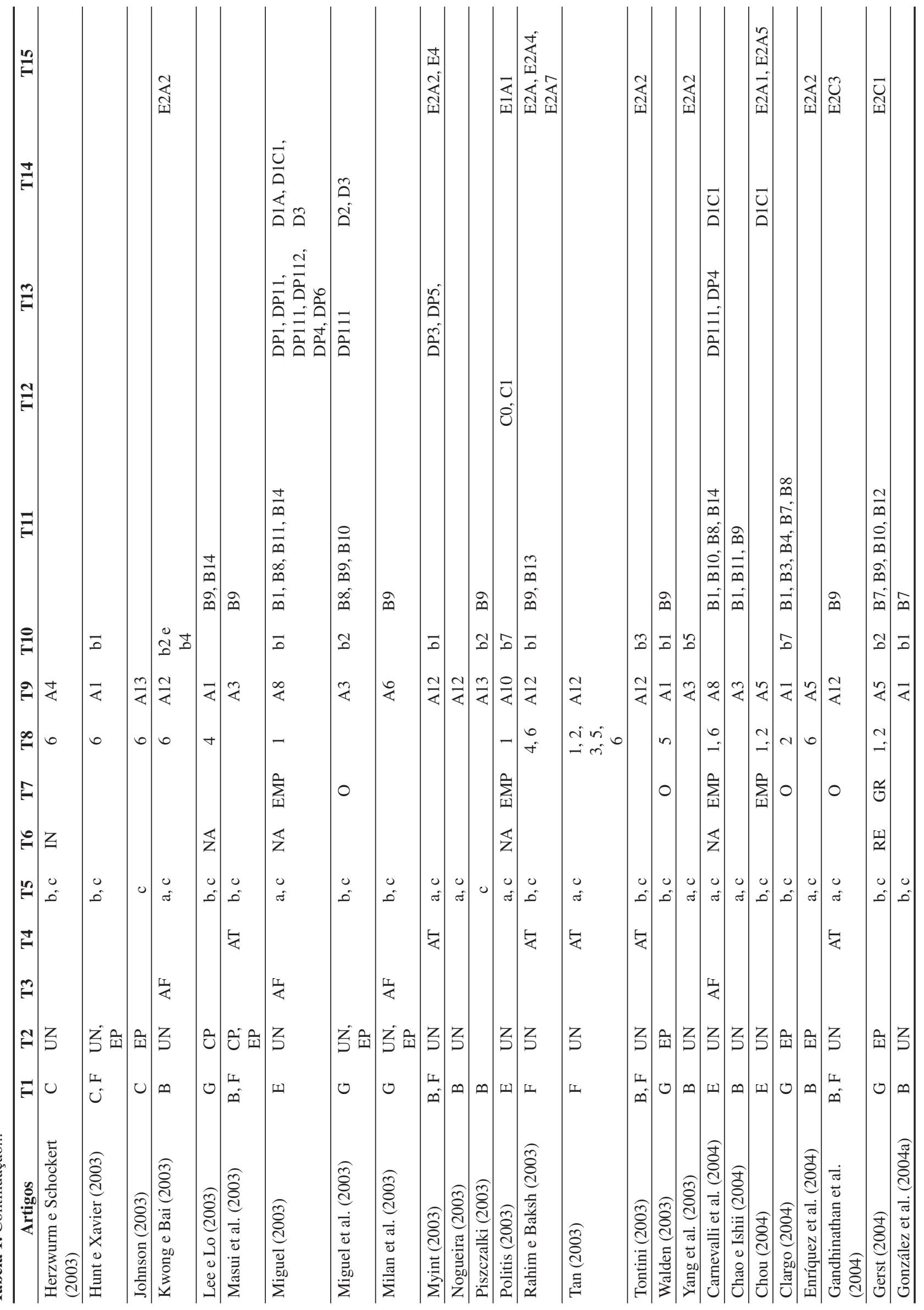




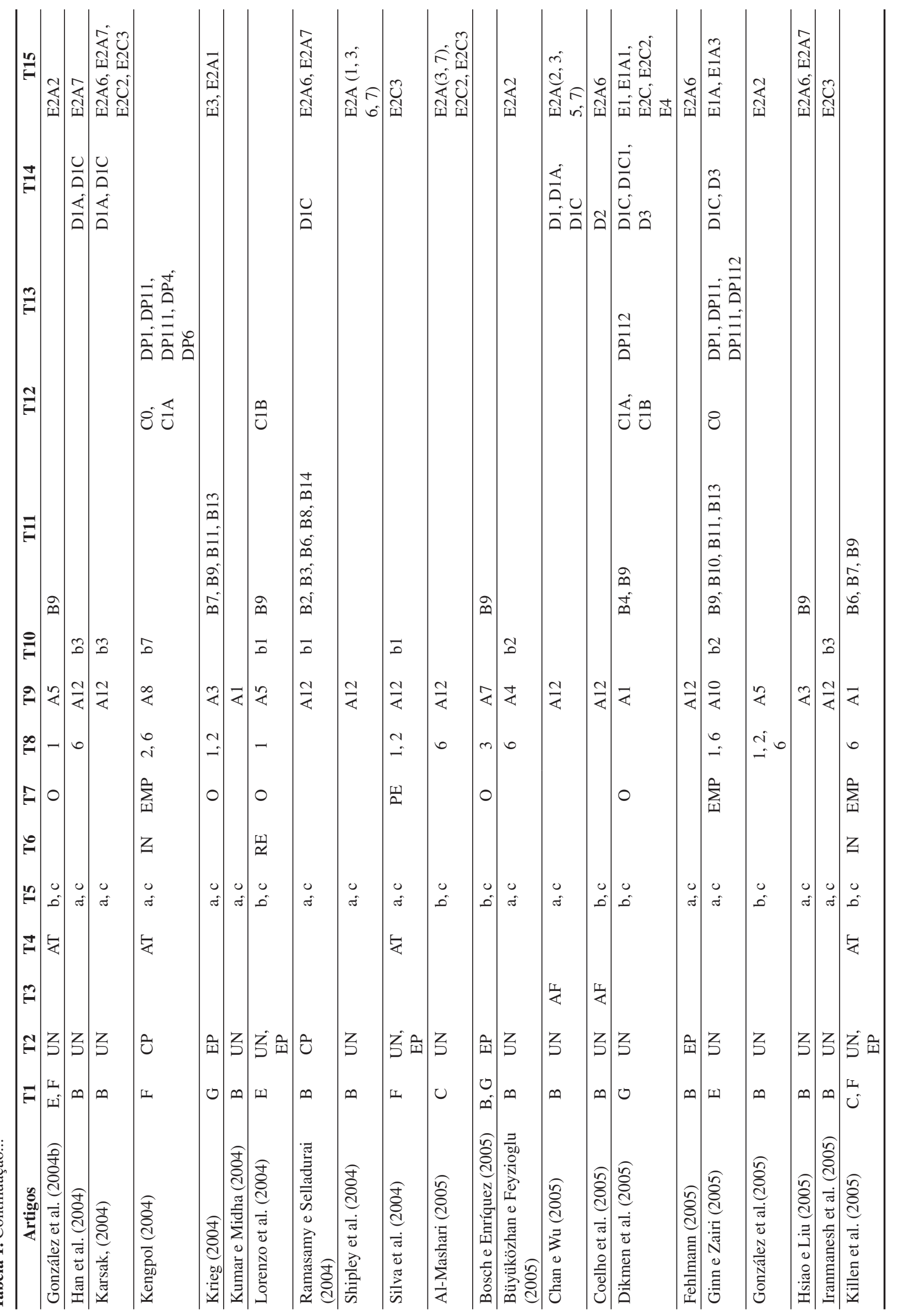




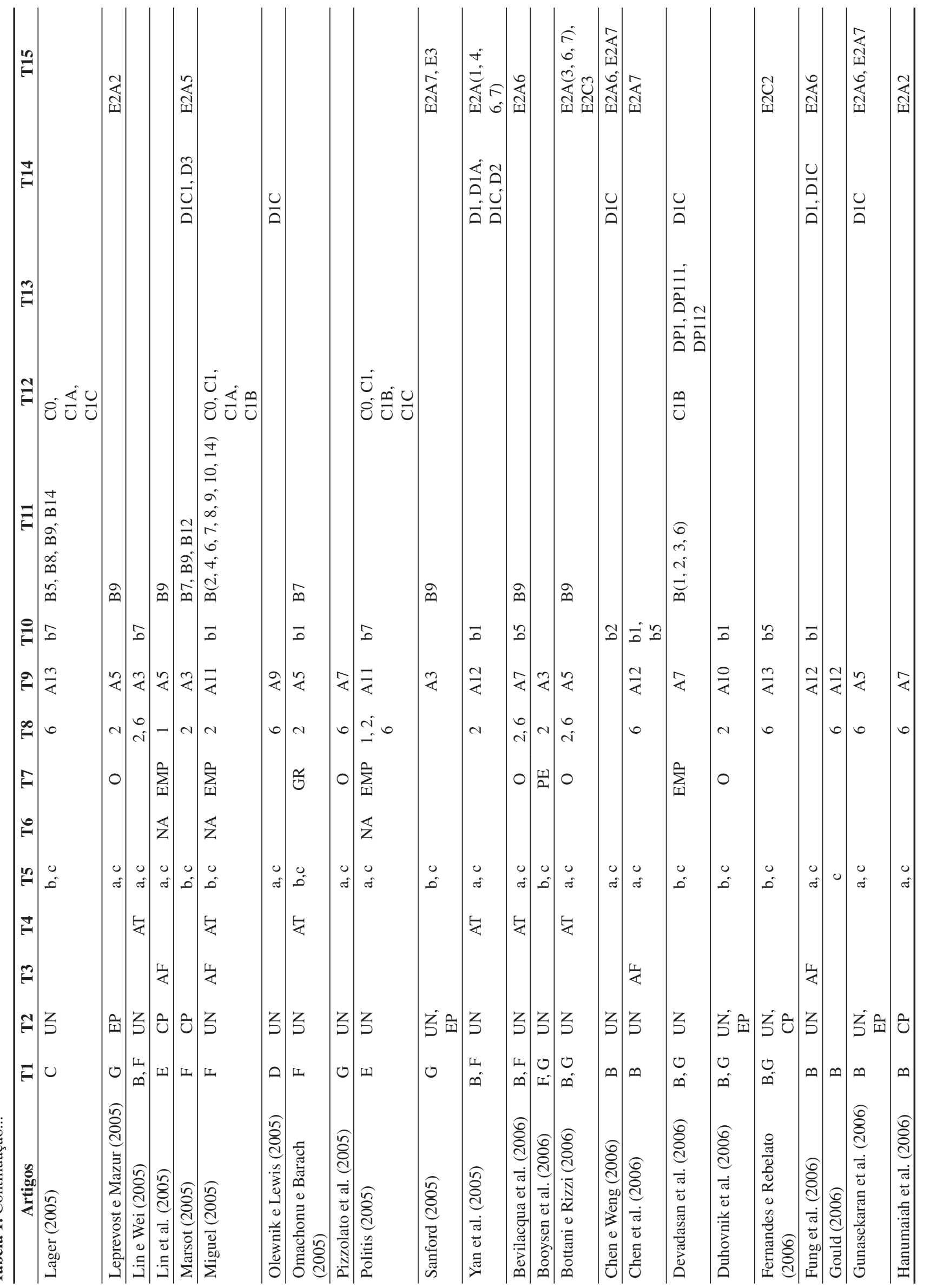




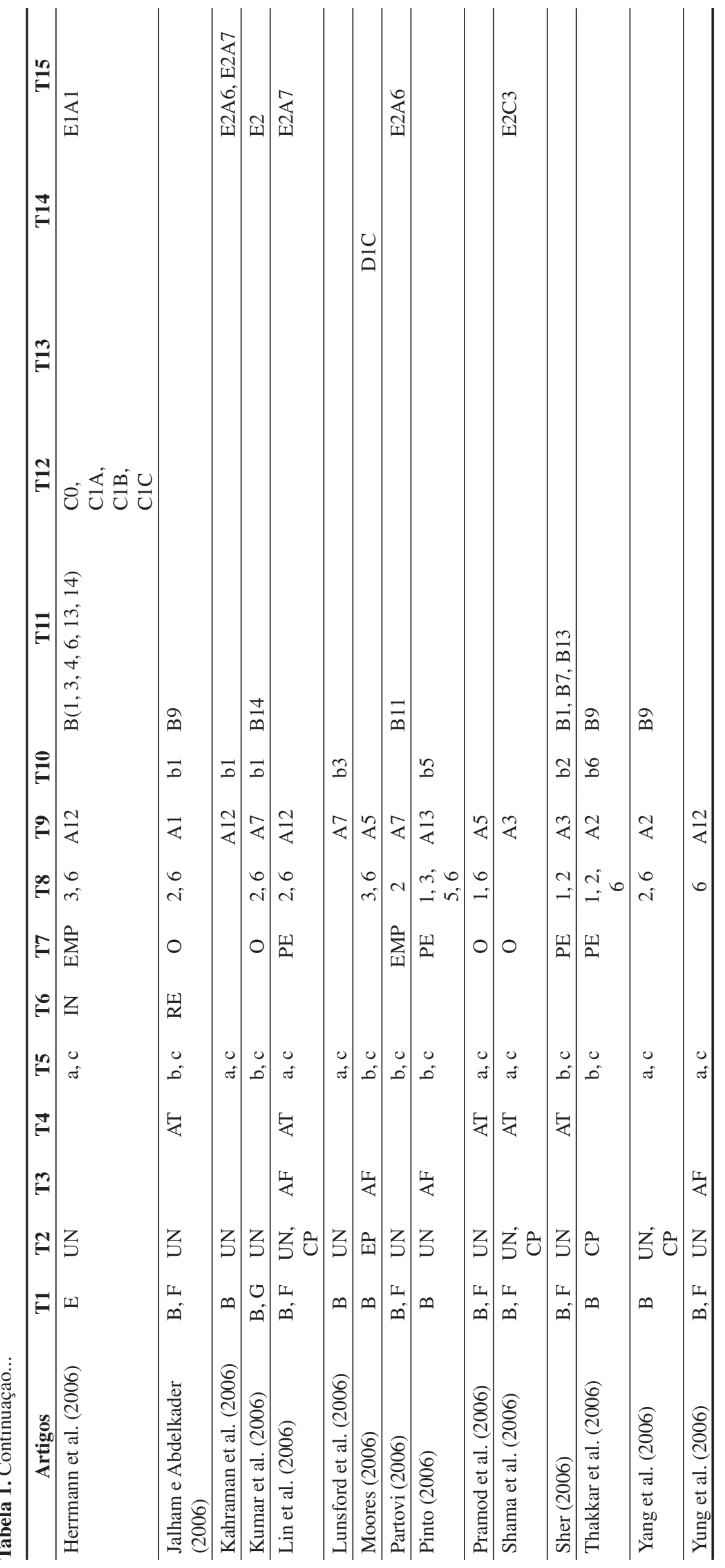




\section{Sobre os autores}

\section{José Antonio Carnevalli}

\section{Paulo Augusto Cauchick Miguel}

Núcleo de Gestão da Qualidade \& Metrologia, Faculdade de Engenharia, Arquitetura e Urbanismo,

Universidade Metodista de Piracicaba - UNIMEP,

Rod. SP 306, Km 1, CEP 13450-000, Santa Bárbara d' Oeste, SP,

e-mails: jcarnevalli@ hotmail.com; paulo.miguel@poli.usp.br

Agradecimentos: Os autores agradecem à CAPES pelo apoio recebido (bolsa de doutorado). 